\title{
BERGLUND-HÜBSCH MIRROR SYMMETRY VIA VERTEX ALGEBRAS
}

\author{
LEV A. BORISOV
}

\begin{abstract}
We give a vertex algebra proof of the Berglund-Hübsch duality of nondegenerate invertible potentials. We suggest a way to unify it with the Batyrev-Borisov duality of reflexive Gorenstein cones.
\end{abstract}

\section{INTRODUCTION}

Ever since its been first discovered in the early 1990's, mirror symmetry served as an inspiration to algebraic and symplectic geometers. The original physical motivation behind it centers around the notion of $N=(2,2)$ superconformal field theory, which is a very rich and only partially axiomatized structure. There are physical methods of assigning such theories to various combinatorial and/or geometric data. In certain instances, the theories one obtains from two different sets of data are isomorphic via a very special mirror involution, which leads to a deep connection between the two sets of data. The earliest example was the prediction of the (virtual) number of rational curves of given degree on a generic quintic threefold [CaOGP].

Most classical treatments of mirror symmetry revolve around the calculation of the so-called $A$ and $B$ chiral rings which are particular substructures of $N=(2,2)$ superconformal field theory. A mirror set of data is characterized by the property that the two rings are interchanged. The $A$ ring of one set of data is supposed to be isomorphic to the $B$ ring of the mirror set of data and vice versa. In many cases these two rings can be constructed mathematically from the initial data, even while the whole theory can only be constructed physically. For example, one can start with a Calabi-Yau manifold $X$ with a complexified Kähler class $[w]$. Then the $A$ ring is the quantum cohomology of $X$ with the parameters specialized to $[w]$. The $B$ ring is the cohomology of the exterior algebra of the tangent bundle of $X$.

In the examples of interest, the $A$ and $B$ rings of the theory come with a double grading and with a natural vector space isomorphism between them. If $\hat{c}>0$ is the central charge of the theory (equal to the 
dimension of $X$ in the above example), then this isomorphism sends a $(p, q)$-graded piece of the $A$ ring to the $(\hat{c}-p, q)$ graded piece of the $B$ ring. Of course, this isomorphism is not compatible with the product structures.

Mirror symmetry construction of Batyrev Ba2 and its generalization to Calabi-Yau complete intersections in toric varieties known as Batyrev-Borisov construction is one the best understood settings of mirror symmetry. In contrast, the Berglund-Hübsch construction of [BeH] has unfortunately received too little attention, despite its appealing simplicity. Recently, papers of Krawitz Kra and Chiodo-Ruan $\mathrm{ChR}$ partially corrected this injustice by proving the conjectural mirror symmetry of Berglund-Hübsch at the level of double graded dimensions, and by connecting the Landau-Ginzburg version of the theory to the geometric notion of orbifold cohomology. Importantly, Krawitz was able to define the dual potential and group of Berglund-Hübsch construction in full generality, beyond the original examples of Berglind and Hübsch. This paper hopes to further advance the understanding of Berglund-Hübsch-Krawitz duality and to also sketch a path that will likely lead to a combined setting that includes both Batyrev-Borisov and Berglund-Hübsch constructions as special cases. Specifically, we find that the vertex algebra approach to mirror symmetry, originally developed for Batyrev-Borisov setting in [Bo2] and [Bo3] can be applied with some modifications to the Berglund-Hübsch setting. It allows us to reprove the result of [Kra and moreover to show the ring isomorphism between the $A$ ring of a Berglund-Hübsch potential and the $B$ ring of the dual potential (with the appropriate choices of orbifoldizations). In the process it leads to a natural combinatorial setup for the unification of the two constructions.

The vertex algebra approach to mirror symmetry aims to construct a larger algebraic structure which contains the $A$ and $B$ rings of the theory as two subspaces, with the induced structure of supercommutative double graded rings. The mirror set of data gives the same larger structure, up to a natural involution that has the effect of switching $A$ and $B$ rings. Specifically, this larger algebraic structure is known as vertex (in some treatments chiral) algebra with $N=2$ structure, and the $A$ and $B$ rings are the chiral rings of this algebra, in the sense of [LeVW]. From the physics view point this vertex algebra is the state space of the half-twisted theory. We recall the definition of these algebras in Section 4. The aforementioned isomorphism of $A$ and $B$ rings of the same theory comes from a natural physical construction called spectral flow. The original calculations of [Bo2] have been heavily inspired 
by the chiral de Rham complex construction of Malikov, Schechtman and Vaintrob, see [MSV]. However, the setting of this paper is more combinatorial, since the underlying geometry is often not clear.

The structure of the paper is as follows. In Section 2 we recall the definitions of Berglund-Hübsch potentials, following [Kra, and then rephrase the construction in the terms that are similar to those of Batyrev-Borisov duality. Specifically, we encode a pair of dual potentials and groups $(W, G)$ and $\left(W^{\vee}, G^{\vee}\right)$ by a pair of dual lattices $M$ and $N$ and collections of elements $\Delta$ and $\Delta^{\vee}$ in these lattices. The pairings of elements of $\Delta$ and $\Delta^{v}$ encode the matrix of degrees that occurs in the definition of Berglund-Hübsch potentials. The elements of $\Delta$ and the elements of $\Delta^{\vee}$ generate cones $K_{M}$ and $K_{N}$ respectively. While $K_{M}$ and $K_{N}$ are not quite dual to each other (as would be the case in Batyrev-Borisov setting) they are close to being dual, because of the nondegeneracy of the potentials.

In Section 3 we give a novel description of $A$ and $B$ rings of BerglundHübsch construction by what essentially amounts to a switch to logarithmic coordinates. We describe the $A$ and $B$ rings of $(W, G)$ as cohomology of the following complex, which is analogous to the one in [BoM].

Proposition 3.3.6. The $A$ ring of Berglund-Hübsch construction, with components twisted by certain one-dimensional spaces, is naturally isomorphic to the cohomology of

$$
\mathbb{C}\left[\left(K_{N}^{\vee} \oplus K_{N}\right)_{0}\right] \otimes \Lambda^{*}\left(M_{\mathbb{C}}\right)
$$

with respect to

$$
d^{A}:=\sum_{m \in \Delta}[m] \otimes(\wedge m)+\sum_{n \in \Delta \vee}[n] \otimes(\text { contr. } n) .
$$

Here $\mathbb{C}\left[\left(K_{N}^{\vee} \oplus K_{N}\right)_{0}\right]$ is the quotient of the semigroup ring of $K_{N}^{\vee} \oplus K_{N}$ by the ideal generated by monomials $m \oplus n$ with $m \cdot n>0$. The $B$ ring is similarly described in Proposition 3.3.2 by replacing $\Lambda^{*}\left(M_{\mathbb{C}}\right)$ $\Lambda^{*}\left(N_{\mathbb{C}}\right)$ and switching contractions and exterior multiplications. We also observe that the natural double grading on these spaces, which was first considered in $[\mathrm{BoM}$ coincides with the double grading of $A$ and $B$ rings described in [Kra], following the more general work of Kaufmann [Kau3]. In the language of Section [3, the difference between the $A$ ring of $(W, G)$ and the $B$ ring of $\left(W^{\vee}, G^{\vee}\right)$ amounts to a switch from $\mathbb{C}\left[\left(K_{N}^{\vee} \oplus K_{N}\right)_{0}\right]$ to $\mathbb{C}\left[\left(K_{M} \oplus K_{M}^{\vee}\right)_{0}\right]$. While Sections 2 and 3 are free of any vertex algebra techniques and are rather elementary, we 
will need the full machinery of vertex algebras to construct a natural isomorphism between these spaces.

In Section 4 we give a brief review of vertex algebras and $N=2$ structures. We also recall lattice vertex algebras Fock $_{M \oplus N}$ constructed from pairs of dual lattices $M$ and $N$.

In Section 5 we define the vertex algebras of Berglund-Hübsch mirror symmetry and prove their first properties. Specifically, for a pair $(f, g)$ of generic coefficient functions $f: \Delta \rightarrow \mathbb{C}$ and $g: \Delta^{\vee} \rightarrow \mathbb{C}$ we define $V_{f, g}$ to be the cohomology of the lattice vertex algebra Fock ${ }_{M \oplus N}$ by the differential

$D_{f, g}:=\operatorname{Res}_{z=0}\left(\sum_{m \in \Delta} f(m) m^{f e r m}(z) \mathrm{e}^{\int m^{b o s}(z)}+\sum_{n \in \Delta^{\vee}} g(n) n^{f e r m}(z) \mathrm{e}^{\int n^{b o s}(z)}\right)$

We prove an analog of the Key Lemma of Bo2 which states that Fock $_{M \oplus N}$ can be replaced by Fock $_{K_{M} \oplus N}$ or Fock $_{M \oplus K_{N}}$. This is a nontrivial consequence of the nondegeneracy of the potential, and it is absolutely crucial for the subsequent discussion.

In Section 6 we use the results of Section 5 and [Bo3] to show that each of the chiral rings of $V_{f, g}$ is naturally isomorphic to the cohomology of a pair of complexes. When $(f, g)=(\mathbf{1}, \mathbf{1})$ these complexes are precisely the ones considered in Section 3, which shows the isomorphism between the $A$ and $B$ rings of the dual Berglund-Hübsch potentials. The main result of the paper is Theorem 6.2.1.

Theorem 6.2.1. For generic choices of $f$ and $g$ the $N=2$ vertex algebra $D_{f, g}$ is of $\sigma$-model type. In particular, this algebra is of $\sigma$ model type for $(f, g)=(\mathbf{1}, \mathbf{1})$. The $B$ ring of $V_{\mathbf{1 , 1}}$ can be identified with the $B$ ring of $(W, G)$ and with the $A \operatorname{ring}$ of $\left(W^{\vee}, G^{\vee}\right)$. The $A$ ring of $V_{\mathbf{1}, \mathbf{1}}$ can be identified with the $A \operatorname{ring}$ of $(W, G)$ and with the $B$ ring of $\left(W^{\vee}, G^{\vee}\right)$.

One advantage of our method, as compared to that of [Kra] is that it does not make explicit use of the classification of invertible potentials of Kreuzer and Skarke [KreS1]. This does not make our argument easier, given the extensive use of the vertex algebra techniques. However, it does make our approach more natural and it allows us to attempt to unify Berglund-Hübsch and Batyrev-Borisov constructions by assuming sufficient conditions that ensure that the argument of Sections 5 and 6 is stil applicable. This unification is explored in Section 7 . The condition is a generalization of nondegeneracy of the potential to the case when the number of elements in $\Delta$ and $\Delta^{\vee}$ is larger than the rank of the lattice. We give two equivalent formulations in Definition 7.1.1 
and Proposition [7.1.3. Finally, in Section 8 we state several natural open problems.

The reader should be forewarned regarding our notation choices. We deliberately use the notations for Berglund-Hübsch setting that are analogous to the Batyrev-Borisov setting. Eventually, the two pictures are joined in Section [7, but until that point we try to make it clear from the context which setting we are considering.

Acknoledgements. I would like to thank Max Kreuzer for introducing me to the Berglund-Hübsch construction and for useful comments on the first version of the paper. The preprints [ChR and Kra were also a major influence. I would like to thank Ralph Kaufmann for useful comments on the first version of the paper. This work was partially supported by the NSF Grant 1003445.

\section{Berglund-HÜBsch AND BAtyrev-Borisov CONSTRUCTIONS}

The goal of this section is to restate the Berglund-Hübsch construction of mirror potentials in the terms that are similar to Batyrev's reflexive polyhedra construction and, more generally, to Batyrev-Borisov dual reflexive cones construction.

2.1. Berglund-Hübsch potentials. The mirror symmetry construction of Berglund and Hübsch begins with a nondegenerate polynomial potential

$$
W\left(x_{1}, \ldots, x_{d}\right)=\sum_{i=1}^{d} c_{i} \prod_{j=1}^{d} x_{j}^{a_{i j}}
$$

with invertible integer matrix $\left(a_{i j}\right)$, where the coefficients $c_{i}$ can be assumed to equal 1 without loss of generality. The variables $x_{j}$ are assumed to be given positive rational degrees $q_{j}$ which make $W$ homogeneous of degree 1, i.e. we have

$$
\sum_{j=1}^{d} a_{i j} q_{j}=1
$$

for all $i$. Nondegeneracy of the potential means that the hypersurface $W=0$ in $\mathbb{C}^{d}$ is smooth away from the origin. It is a very restrictive condition, and complete classification of nondegenerous potentials is given in [KreS1].

Consider the group $\operatorname{Aut}(W)$ of diagonal automorphisms

$$
\gamma: x_{j} \mapsto \gamma_{j} x_{j}
$$


which preserve the potential $W$. This is a finite abelian group, which contains two natural subgroups. First, $\operatorname{SL}_{d} \cap \operatorname{Aut}(W) \subseteq \operatorname{Aut}(W)$ is characterized by $\prod_{j} \gamma_{j}=1$. Second, there is a subgroup of $\operatorname{Aut}(W)$ generated by the automorphism

$$
x_{j} \mapsto \exp \left(2 \pi \mathrm{i} q_{j}\right) x_{j}
$$

which is called the exponential grading operator in [Kra]. We will be mostly interested in the case when the second of these groups is contained in the first one, which means that the exponential graded operator acts with determinant one. This translates into

$$
\sum_{j=1}^{d} q_{j}=k \in \mathbb{Z}_{>0} .
$$

Remark 2.1.1. Condition (2) is a generalization of the Calabi-Yau condition $\sum_{j=1}^{d} q_{j}=1$, see $\mathrm{ChR}$. The case $\sum_{j=1}^{d} q_{j}=k$ will turn out to be analogous to Batyrev-Borisov construction for Calabi-Yau complete intersections of $k$ hypersurfaces. We will thus continue to refer to the above condition as the Calabi-Yau condition.

As part of the combinatorial data of Berglund-Hübsch construction one fixes a subgroup $G \subseteq \operatorname{Aut}(W)$.

2.2. Dual potential and dual group. It follows from the classification of [KreS1] that if

$$
W=\sum_{i=1}^{d} \prod_{j=1}^{d} x_{j}^{a_{i j}}
$$

is a nondegenerate potential, then

$$
W^{\vee}=\sum_{i=1}^{d} \prod_{j=1}^{d} y_{j}^{a_{j i}}
$$

is also nondegenerate.

Let $G$ be a subgroup of $\operatorname{Aut}(W)$. Then Krawitz [Kra] defines the dual group $G^{\vee} \subseteq \operatorname{Aut}\left(W^{\vee}\right)$. We describe his construction while trying to keep our notations as compatible as possible. Define $A_{W}=\left(a_{i j}\right)$. Then consider the inverse matrix $A_{W}^{-1}$ and define $\rho_{i}$ and $\bar{\rho}_{i}$ to be its $i$ th column and $i$-th row respectively. Abusing the notation slightly, we will also denote by $\rho_{i}$ the corresponding element of $\operatorname{Aut}(W)$ obtained by multiplying $x_{j}$ by $\exp \left(2 \pi \mathrm{i}\left(\rho_{i}\right)_{j}\right)$. Similarly, $\bar{\rho}_{j}$ will also be used to denote an element of $\operatorname{Aut}\left(W^{\vee}\right)$. 
Definition 2.2.1. Kra The dual group $G^{\vee}$ is defined by

$$
G^{\vee}:=\left\{\prod_{i=1}^{d} \bar{\rho}_{i}^{r_{i}} \mid\left[r_{1}, \cdots, r_{d}\right] A_{W}^{-1}\left[\begin{array}{c}
a_{1} \\
\vdots \\
a_{d}
\end{array}\right] \in \mathbb{Z} \text { for all } \prod_{i=1}^{d} \rho_{i}^{a_{i}} \in G\right\} .
$$

2.3. Combinatorial reformulation of the Berglund-Hübsch potential and group. We will now reformulate the data of BerglundHübsch potential $W$ and the group $G$ in combinatorial terms that resemble the setting of Batyrev-Borisov mirror symmetry construction.

Consider the free abelian groups $M_{0}$ and $N_{0}$ with bases $\left(u_{i}\right), i=$ $1, \ldots, d$ and $\left(v_{j}\right), j=1, \ldots, d$. Define an integer, nondegenerate (but not unimodular) pairing on these lattices by

$$
u_{i} \cdot v_{j}:=a_{i j}
$$

where $a_{i j}$ are the exponents of the potential $W$, as in the previous subsection.

Proposition 2.3.1. The choice of the group $G$ and its dual $G^{\vee}$ is naturally equivalent to the choice of suplattices $M \supseteq M_{0}$ and $N \supseteq N_{0}$ such that the induced pairing on $M$ and $N$ is integer and unimodular, i.e. $M$ and $N$ are dual lattices.

Proof. First observe that $\operatorname{Aut}(W)$ is naturally isomorphic to $M_{0}^{\vee} / N_{0}$. The isomorphism sends $\gamma=\left(\gamma_{i}\right)$ in (11) to $N_{0}+\sum_{j=1}^{d} \frac{1}{2 \pi \mathrm{i}}\left(\log \gamma_{j}\right) v_{j}$. Consequently, a choice of $G$ means a choice of lattice $N$ with $N_{0} \subseteq$ $N \subseteq M_{0}^{\vee}$. We have a similar identification of $\operatorname{Aut}\left(W^{\vee}\right)$ with $N_{0}^{\vee} / M_{0}$. It remains to show that the definition of $G^{\vee}$ in the previous subsection is equivalent to considering $M=N^{\vee}$. Indeed, $\rho_{i}$ correspond to the elements of the basis of $M_{0}^{\vee}$ which is dual to the basis $\left(e_{i}\right)$ of $M$ and $\bar{\rho}_{j}$ correspond to the basis of $N_{0}^{\vee}$ which is dual to the basis $\left(v_{j}\right)$ of $N$. The pairing matrix $\left(\bar{\rho}_{i} \cdot \rho_{j}\right)$ is equal by $A_{W}^{-1}$ and the rest is clear.

Remark 2.3.2. The Berglund-Hübsch duality now simply means interchanging the two lattices $M$ and $N$ together with the sets of chosen elements $\left(u_{i}\right),\left(v_{j}\right)$ in them. It is now obvious that $\left(G^{\vee}\right)^{\vee}=G$.

We can now give a simple combinatorial interpretation of the two subgroups of $\operatorname{Aut}(W)$ and the Calabi-Yau condition.

Definition 2.3.3. We define elements $\operatorname{deg} \in N_{0}^{\vee}$ and $\operatorname{deg}^{\vee} \in M_{0}^{\vee}$ by $u_{i} \cdot \operatorname{deg}{ }^{\vee}=1, \operatorname{deg} \cdot v_{i}=1$, for all $i$.

In the following proposition we implicitly assume the identification of the data of $G$ and $G^{\vee}$ with the dual suplattices $M$ and $N$ in Proposition 2.3 .1 . 
Proposition 2.3.4. The group $G$ lies in $\mathrm{SL}_{d} \cap \operatorname{Aut}(W)$ if and only if deg $\in M$. The group $G$ contains the exponential grading operator if and only if $\operatorname{deg}^{\vee} \in N$. The Calabi-Yau condition is equivalent to $\operatorname{deg} \cdot \operatorname{deg}{ }^{\vee} \in Z_{>0}$.

Proof. The condition that $\operatorname{deg} \in M$ is equivalent to the statement that deg has integer pairing with every $\sum_{j=1}^{d} \frac{1}{2 \pi \mathrm{i}}\left(\log \gamma_{j}\right) v_{j}$ for $\gamma=\left(\gamma_{i}\right) \in G$. This pairing is $\sum_{j=1}^{d} \frac{1}{2 \pi \mathrm{i}}\left(\log \gamma_{j}\right)$, and its integrality is equivalent to $\prod_{j} \gamma_{j}=1$. The element $\operatorname{deg}^{\vee}$ equals $\sum_{j} q_{j} v_{j}$, which in turns corresponds to the exponential grading operator. Finally, $\operatorname{deg} \cdot \operatorname{deg}{ }^{\vee}=$ $\operatorname{deg} \cdot \sum_{j} q_{j} v_{j}=\sum_{j} q_{j}$

Corollary 2.3.5. The group $G$ lies in $S L_{d}$ if and only if $G^{\vee}$ contains the exponential grading operator. The Calabi-Yau condition means that there exists $G$ such that the corresponding lattices $M$ and $N$ satisfy both $\operatorname{deg} \in M$ and $\operatorname{deg}^{\vee} \in N$.

Proof. Clear.

Remark 2.3.6. In what follows we will be mostly interested in the case when the group $G$ contains the exponential grading operator and lies in $\mathrm{SL}_{d}$. In particular, the potential $W$ must satisfy the CalabiYau condition. We will refer to this situation as Berglund-Hübsch construction of Calabi-Yau type of index $k$ where $k=\sum_{j} q_{j}$.

We introduce the following notations to highlight the analogy between the combinatorial data of Berglund-Hübsch and Batyrev-Borisov constructions.

Definition 2.3.7. Define the cones $K_{M}$ in $M$ and $K_{N}$ in $N$ by

$$
K_{M}:=\sum_{i} \mathbb{Q}_{\geq 0} u_{i}, K_{N}:=\sum_{j} \mathbb{Q}_{\geq 0} v_{j}
$$

Proposition 2.3.8. Consider the dual cones $K_{M}^{\vee}$ in $N$ and $K_{N}^{\vee}$ in $M$. Then we have $K_{M} \subseteq K_{N}^{\vee}$ and $K_{N} \subseteq K_{M}^{\vee}$.

Proof. The statement follows from $a_{i j} \geq 0$.

Remark 2.3.9. In the case of Fermat type potential (see [KreS1]), we have equalities in Proposition 2.3.8. In general, the inclusions are strict. However, the nondegeneracy of the polynomial potential $W$ may be viewed as the statement that the complements are in some sense small.

Definition 2.3.10. Denote by $\Delta$ the set $\left(u_{i}\right)$ and by $\Delta^{\vee}$ the set $\left(v_{j}\right)$. 
Remark 2.3.11. We may associate arbitrary nonzero elements $c_{i}$ to the elements $u_{i} \in \Delta$, as in the original definition of $W$. Similarly, we can associate arbitrary elements to monomials in $W^{\vee}$. These can all be rescaled to 1 .

2.4. Comparison with Batyrev-Borisov construction. Recall that the discrete combinatorial data of Batyrev-Borisov construction consist of the pair of dual lattices $M$ and $N$ and dual reflexive Gorenstein cones $K$ in $M$ and $K^{\vee}$ in $N$.

Definition 2.4.1. Let $M$ and $N$ be dual lattices. The dual rational polyhedral cones $K$ in $M$ and $K^{\vee}$ in $N$ are called dual Gorenstein if there exist elements $\operatorname{deg} \in M$ and $\operatorname{deg}^{\vee} \in N$ so that the lattice generators $u$ of the rays of $K$ satisfy $u \cdot \mathrm{deg}^{\vee}=1$ and lattice generators $v$ of rays of $K^{\vee} \operatorname{satisfy~} \operatorname{deg} \cdot v=1$.

Definition 2.4.2. [Bo3] Define $\Delta$ to be the set of lattice elements $m \in K$ which satisfy $m \cdot \operatorname{deg}^{\vee}=1$ and $\Delta^{\vee}$ to be the set of lattice elements $n \in K^{\vee}$ such that $\operatorname{deg} \cdot n=1$.

Definition 2.4.3. The index of the pair of dual reflexive Gorenstein cones is defined as deg $\cdot \mathrm{deg}^{\vee}$.

Remark 2.4.4. The case of index one corresponds to the original Batyrev duality, see Ba2]. The higher index $k$ case may or may not have a geometric underpinning. In good cases, one side of the duality may be related to a Calabi-Yau complete intersection of $k$ hypersurfaces. In the best case scenario, both sides are related to a complete intersection. This best case scenario corresponds to the nef-partition case, considered in [Bo1]. The issue is whether deg (resp. $\operatorname{deg}^{\vee}$ ) lie in the semigroup generated by $\Delta$ (resp. $\Delta^{\vee}$ ). The reader is referred for more details to the paper of Batyrev and Nill [BaN].

Another important ingredient of Batyrev-Borisov construction is a pair of coefficient functions, which are generic functions $f: \Delta \rightarrow \mathbb{C}$ and $g: \Delta^{\vee} \rightarrow \mathbb{C}$. In the good cases they correspond to the coefficients of the defining equations of a pair of elements of mirror families. BatyrevBorisov duality corresponds to simply switching all the data and their duals.

It should now be clear to the reader that the above formulation of Berglund-Hübsch construction of Calabi-Yau type of index $k$ almost fits into the framework of Batyrev-Borisov construction of index $k$. Specifically, we have dual lattices $M$ and $N$, elements $\operatorname{deg} \in M$ and $\operatorname{deg}^{\vee} \in N$, finite sets $\Delta$ and $\Delta^{\vee}$, with $\Delta \cdot \operatorname{deg}^{\vee}=1, \operatorname{deg} \cdot \Delta^{\vee}=1$, deg $\cdot \operatorname{deg}^{\vee}=k$, as well as generic coefficient functions $f, g$. The only difference is 
that in the Berglund-Hübsch case the cones $K_{M}=\sum_{m \in \Delta} \mathbb{Q}_{\geq 0} m$ and $K_{N}=\sum_{n \in \Delta \vee} \mathbb{Q}_{\geq 0} n$ are not quite dual to each other but only satisfy $K_{M} \cdot K_{N} \geq 0$. In what follows, we will pursue this parallel to its fullest by applying to the Berglund-Hübsch setting the ideas and results of the vertex algebra approach, developed in the context of Batyrev-Borisov mirror construction. Afterwards we will attempt to unify the two constructions in Section 7 .

We will first pursue the analogy of Berglund-Hübsch and BatyrevBorisov constructions at the level of $A$ and $B$ rings, avoiding the vertex algebra machinery. However, we will eventually invoke the full machinery of the vertex algebra approach to prove the Berglund-Hübsch duality.

\section{The DOUBle-GRADED COHOMOLOGY SPACES}

In this section, we reinterpret the $A$ and $B$ rings of Berglund-Hübsch construction as the cohomology of some double-graded complexes. These complexes are motivated by the calculation of $A$ and $B$ rings for BatyrevBorisov construction in [BoM].

3.1. Calculation of $\mathrm{BoM}$ of the $A$ and $B$ rings of BatyrevBorisov construction. Let $M$ and $N$ be dual lattices, $K$ in $M$ and $K^{\vee}$ in $N$ a pair of dual Gorenstein cones, $\operatorname{deg} \in M$, $\operatorname{deg}^{\vee} \in N$ the corresponding degree elements, and $\Delta \subset M$ and $\Delta^{\vee} \subset N$ the degree one elements of the appropriate cones. Let $f$ and $g$ be generic coefficient functions.

Consider the vector space $\mathbb{C}\left[\left(K \oplus K^{\vee}\right)_{0}\right]$ which has a basis indexed by the pairs of lattice points $(m, n)$ in $\left(K, K^{\vee}\right)$ with $m \cdot n=0$. We will denote the corresponding monomials by $[m+n]$. This vector space has a natural structure of the module over the semigroup ring $\mathbb{C}\left[K \oplus K^{\vee}\right]$, since it is the quotient of $\mathbb{C}\left[K \oplus K^{\vee}\right]$ by the ideal generated by $[m+n]$ with $m \cdot n>0$. The following description of the $A$ and $B$ rings of Batyrev-Borisov mirror symmetry construction has been suggested in [BoM] and [Bo3].

Definition 3.1.1. Consider the space

$$
\mathbb{C}\left[\left(K \oplus K^{\vee}\right)_{0}\right] \otimes_{\mathbb{C}} \Lambda^{*}\left(M_{\mathbb{C}}\right)
$$

with endomorphism $d_{f, g}^{A}$ given by

$$
d_{f, g}^{A}:=\sum_{m \in \Delta} f(m)[m] \otimes(\wedge m)+\sum_{n \in \Delta^{\vee}} g(n)[n] \otimes(\text { contr. } n)
$$


where contr. $n$ means taking a contraction in the exterior algebra of $M_{\mathbb{C}}$ by element $n$. The cohomology of $\mathbb{C}\left[\left(K \oplus K^{\vee}\right)_{0}\right] \otimes_{\mathbb{C}} \Lambda^{*}\left(M_{\mathbb{C}}\right)$ with respect to $d_{f, g}^{A}$ is called the $A$ ring associated to the data $\left(M, N, K, K^{\vee}, f, g\right)$. Similarly, the cohomology of

$$
\mathbb{C}\left[\left(K \oplus K^{\vee}\right)_{0}\right] \otimes_{\mathbb{C}} \Lambda^{*}\left(N_{\mathbb{C}}\right)
$$

under

$$
d_{f, g}^{B}:=\sum_{m \in \Delta} f(m)[m] \otimes(\operatorname{contr} . m)+\sum_{n \in \Delta^{\vee}} g(n)[n] \otimes(\wedge n)
$$

is called the $B$ ring associated to these data.

Definition 3.1.2. The $A$ ring possesses a natural double grading as follows. There is the conformal grading induced from the grading on $\mathbb{C}\left[\left(K \oplus K^{\vee}\right)_{0}\right] \otimes_{\mathbb{C}} \Lambda^{*}\left(M_{\mathbb{C}}\right)$ which assigns to $[m \oplus n] \otimes P$ the degree $-m \cdot \operatorname{deg}{ }^{\vee}+\operatorname{deg} \cdot n+\operatorname{deg}(P)-\operatorname{deg} \cdot \operatorname{deg}^{\vee}$. This grading is preserved by $d_{f, g}^{A}$. There is an additional cohomological grading induced by $m$. $\operatorname{deg}{ }^{2}+\operatorname{deg} \cdot n$ which is increased by one by $d_{f, g}^{A}$.

Definition 3.1.3. The $B$ ring possesses a natural double grading as follows. The conformal grading assigns to $[m \oplus n] \otimes P$ in $\mathbb{C}[(K \oplus$ $\left.\left.K^{\vee}\right)_{0}\right] \otimes_{\mathbb{C}} \Lambda^{*}\left(N_{\mathbb{C}}\right)$ the degree $m \cdot \operatorname{deg}^{\vee}-\operatorname{deg} \cdot n+\operatorname{deg}(P)-\operatorname{deg} \cdot \operatorname{deg}{ }^{\vee}$. This grading is preserved by $d_{f, g}^{B}$. The cohomological grading assigns to this element degree $m \cdot \operatorname{deg}^{\vee}+\operatorname{deg} \cdot n-\operatorname{deg} \cdot \operatorname{deg}^{\vee}$. It is increased by one by $d_{f, g}^{B}$.

Remark 3.1.4. The isomorphism between the $\Lambda^{*}\left(M_{\mathbb{C}}\right)$ and $\Lambda^{*}\left(N_{\mathbb{C}}\right)$ induces an isomorphism of the above complexes and thus an isomorphism of $A$ and $B$ rings. This isomorphism clearly preserves the cohomological grading and replaces the conformal grading of $p$ by the conformal grading of $\left(\operatorname{rk} M-2 \mathrm{deg} \cdot \operatorname{deg}^{\vee}\right)-p$. The number $\left(\operatorname{rk} M-2 \operatorname{deg} \cdot \operatorname{deg}^{\vee}\right)$ is the central charge of the theory (and the dimension of the Calabi-Yau variety if one can be associated to this set of data).

The following key proposition has been proved in [BoM].

Proposition 3.1.5. The dimensions of the double-graded components of the A-ring and the B-ring of the theory coincide with the stringy Hodge numbers of the Calabi-Yau variety, if one can be associated to this set of data. In the particular case of $\mathrm{deg} \cdot \mathrm{deg}^{\vee}=1$, this Calabi-Yau variety is $\operatorname{Proj}\left(\mathbb{C}[K] /\left\langle\sum_{m \in \Delta} f(m)[m]\right\rangle\right)$.

It is not at all clear from the above description how to construct a product on the $A$ and $B$ rings above. In fact, the only known general definition invokes the machinery of vertex algebras in [Bo3]. However, 
there are some natural subrings of $A$ and $B$ on which the product can be constructed directly.

Remark 3.1.6. Consider the subspace of

$$
\mathbb{C}\left[\left(K \oplus K^{\vee}\right)_{0}\right] \otimes_{\mathbb{C}} \Lambda^{*}\left(M_{\mathbb{C}}\right)
$$

spanned by the elements of the form $\left[\mathbf{0}+n_{1}\right] \otimes 1$ where $n_{1}$ lies in the interior of $K^{\vee}$. This subspace is annihilated by $d_{f, g}^{A}$, because $m \cdot n_{1}>0$ for all $m \in \Delta$. The image of this space in the $A$ ring is isomorphic, as a $\mathbb{C}$-algebra, to the quotient of $\mathbb{C}\left[K^{\vee}\right] /\left\langle\sum_{n \in \Delta} \vee g(n)(m \cdot n)[n]\right\rangle$ by the annihilator of the element $\left[\mathrm{deg}^{\vee}\right]$. Similarly, there is a natural subring of the $B$ ring which comes from the linear combinations of the elements of the form $\left[m_{1}+\mathbf{0}\right] \otimes 1$ with $m_{1}$ in the interior of $K$.

3.2. Preliminary lemma on Jacobian quotients. In order to give a description of the $A$ and $B$ rings in the Berglund-Hübsch setting, we will first need a simple lemma which applies more generally to any polynomial potential, not necessarily of Berglund-Hübsch type.

Let $q_{i} \in \mathbb{Q}_{>0}$ denote the weights of the variables $x_{i}$ in a polynomial ring of $d$ variables. Let $F\left(x_{1}, \ldots, x_{d}\right)$ be a total degree 1 polynomial such that $F=0$ is smooth away from the origin. Equivalently, the partial derivatives $\frac{\partial F}{\partial x_{i}}$ form a regular sequence. We are interested in the quotient

$$
\mathbb{C}\left[x_{1}, \ldots, x_{d}\right] /\left\langle\frac{\partial F}{\partial x_{1}}, \ldots, \frac{\partial F}{\partial x_{d}}\right\rangle
$$

which we will further shift by the product of the variables. This additional shift is important for finite group actions that need to be considered in the Berglund-Hübsch construction.

We denote by $\mathbb{C}[\mathbf{x}, \mathbf{y}]_{0}$ the quotient of the polynomial ring in $n$ variables by the monomials $x_{i} y_{i}$. It has a basis of monomials whose $x$ support is disjoint from $y$-support. Consider the space

$$
\mathbb{C}[\mathbf{x}, \mathbf{y}]_{0} \otimes \Lambda^{*}\left(\mathbb{C} e_{1}+\ldots+\mathbb{C} e_{d}\right)
$$

and the operator

$$
d_{f, \mathbf{1}}^{B}:=\sum_{i=1}^{d} x_{i} \frac{\partial F}{\partial x_{i}} \otimes \operatorname{contr} . e_{i}^{\vee}+\sum_{i=1}^{d} y_{i} \otimes \wedge e_{i}
$$

on it, where $e_{i}^{\vee}$ is the $i$-th element of the dual basis. 
Lemma 3.2.1. Operator $d_{f, \mathbf{1}}^{B}$ is a differential. Its cohomology is naturally isomorphic to

$$
\left(\prod_{i=1}^{d} x_{i}\right) \mathbb{C}\left[x_{1}, \ldots, x_{d}\right] /\left\langle\frac{\partial F}{\partial x_{1}}, \ldots, \frac{\partial F}{\partial x_{d}}\right\rangle .
$$

Proof. It is easy to see that the terms in $d_{f, \mathbf{1}}^{B}$ anticommute with each other. The only interesting case is the anticommutator of the $i$-th term from the first sum and the $i$-th term from the second sum, which equals $x_{i} y_{i} \frac{\partial F}{\partial x_{i}} \otimes 1$, which is zero because we work in $\mathbb{C}[\mathbf{x}, \mathbf{y}]_{0}$. We can also observe that $d_{f, \mathbf{1}}^{B}$ increases by one the total degree in $\mathbf{x}$ and $\mathbf{y}$ where $x_{i}$ has degree $q_{i}$ and $y_{i}$ has degree 1 .

Consider the subcomplex $\mathcal{C}$ of $\mathbb{C}[\mathbf{x}, \mathbf{y}]_{0} \otimes \Lambda^{*}\left(\mathbb{C} e_{1}+\ldots+\mathbb{C} e_{d}\right)$ which is spanned by elements of the form

$$
P(\mathbf{x}) \prod_{i \in I} x_{i} \otimes\left(\wedge_{i \notin I} e_{i}\right) .
$$

where $I$ ranges over all subsets of $\{1, \ldots, d\}$ and $P$ is any polynomial in $\mathbb{C}\left[x_{1}, \ldots, x_{d}\right]$. The wedge terms of $d_{f, \mathbf{1}}^{B}$ act trivially on it, in view of $x_{i} y_{i}=0$. The contraction terms act exactly like the Koszul complex for $\frac{\partial F}{\partial x_{i}}$ on $\mathbb{C}[\mathbf{x}]$ on the $P$ parts of $(\underline{3})$. As a result, the cohomology of $\mathcal{C}$ is precisely

$$
\left(\prod_{i=1}^{d} x_{i}\right) \mathbb{C}\left[x_{1}, \ldots, x_{d}\right] /\left\langle\frac{\partial F}{\partial x_{1}}, \ldots, \frac{\partial F}{\partial x_{d}}\right\rangle .
$$

It remains to show that

$$
\left(\mathbb{C}[\mathbf{x}, \mathbf{y}]_{0} \otimes \Lambda^{*}\left(\mathbb{C} e_{1}+\ldots+\mathbb{C} e_{d}\right)\right) / \mathcal{C}
$$

has zero cohomology. Consider the double grading given by the degree in $\mathbf{x}$ and the degree in $\mathbf{y}$ where each $y_{i}$ is given degree 1 . Note that the first and second sums $d_{1}$ and $d_{2}$ in $d_{f, \mathbf{1}}^{B}$ change this bidegree by $(1,0)$ and $(0,1)$ respectively. The stupid filtrations converge, because the dimensions of the graded pieces are finite. Thus it suffices to check that cohomology of (44) with respect to $d_{2}$ vanishes.

The cohomology of (44) with respect to $d_{2}$ can be computed separately for each multidegree in $\mathbf{x}$. Consider $\prod_{i \in I} x_{i}^{r_{i}}$ with positive $r_{i}$. The corresponding part of (4) is

$$
\left(\mathbb{C}\left[y_{i}, i \notin I\right] \otimes \Lambda^{*}\left(\oplus_{i=1}^{d} \mathbb{C} e_{i}\right)\right) /\left(\Lambda_{j \notin I} e_{j} \wedge \Lambda^{*}\left(\oplus_{i \in I} \mathbb{C} e_{i}\right)\right) .
$$

This complex is quasi-isomorphic to the tensor product of $\Lambda^{*}\left(\oplus_{i \in I} \mathbb{C} e_{i}\right)$ with trivial differential and the augmented Koszul complex for $\mathbb{C}\left[y_{i}, i \notin\right.$ 
$I]$ and the sequence $\left(y_{i}, i \notin I\right)$. The latter is acyclic, which finishes the proof.

We will now consider abelian group actions in the context of Lemma 3.2.1. Let $G$ be a finite abelian group which acts on $\mathbf{x}$ diagonally and fixes the potential $F$. Consider two dual lattices $\mathbb{Z}^{d}$ with the standard cones $\left(\mathbb{Z}_{\geq 0}\right)^{d}$ such that the corresponding semigroup algebras are identified with $\mathbb{C}\left[x_{1}, \ldots, x_{d}\right]$ and $\mathbb{C}\left[y_{1}, \ldots, y_{d}\right]$ respectively. Let $M$ be the sublattice that consists of degrees of monomials in $\mathbf{x}$ that are fixed by $G$. Denote by $C$ the cone in $M$ which is the intersection of the standard cone with it. Denote by $\mathbb{C}\left[\left(C \oplus\left(\mathbb{Z}_{\geq 0}\right)^{d}\right)_{0}\right]$ the quotient of $\mathbb{C}\left[C \oplus\left(\mathbb{Z}_{\geq 0}\right)^{d}\right]$ by the monomials with positive pairing.

Proposition 3.2.2. The G-fixed part of the (shifted) Milnor ring of $F$ is given by the cohomology of

$$
\mathbb{C}\left[\left(C \oplus\left(\mathbb{Z}_{\geq 0}\right)^{d}\right)_{0}\right] \otimes \Lambda^{*}\left(\oplus_{i=1}^{d} \mathbb{C} e_{i}\right)
$$

with respect to

$$
d_{f, \mathbf{1}}^{B}:=\sum_{i=1}^{d} x_{i} \frac{\partial F}{\partial x_{i}} \otimes \operatorname{contr} . e_{i}^{\vee}+\sum_{i=1}^{d} y_{i} \otimes \wedge e_{i} .
$$

Proof. We first remark that the logarithmic partial derivatives $x_{i} \frac{\partial F}{\partial x_{i}}$ make sense as elements of $\mathbb{C}[C]$. The action of $G$ on the shifted Milnor ring is induced from the diagonal action on $x_{i}$. We consider the trivial action of $G$ on $y_{i}$ and $e_{i}$ to define the action on the complex of Lemma 3.2.1, which induces the same action on the cohomology. It remains to observe that $\mathbb{C}\left[\left(C \oplus\left(\mathbb{Z}_{\geq 0}\right)^{d}\right)_{0}\right]$ is the $G$-invariant part of $\mathbb{C}[\mathbf{x}, \mathbf{y}]_{0}$.

3.3. Calculation of the $A$ and $B$ rings of Berglund-Hübsch construction. We use the notations $W, G, M, N, K_{M}, K_{N}$, deg, deg ${ }^{\vee}$, $\Delta, \Delta^{\vee}$ from Subsection 2.3. In this section we are only concerned with the double-graded vector spaces, so the ring structure is ignored.

The following definition of the $B$ ring of Berglund-Hübsch construction follows from the definitions of Kra].

Definition 3.3.1. The $B$ ring is given by

$$
\left(\oplus_{g \in G} \mathcal{L}_{g}\right)^{G}
$$

where $\mathcal{L}_{g}^{G}$ is the $G$-invariant part of the Milnor ring in the variables $x_{i}$ that are fixed by $g$, with respect to the restriction of the potential, shifted by the product of variables fixed under $g$. 
We define $\mathbb{C}\left[\left(K_{N}^{\vee} \oplus K_{N}\right)_{0}\right]$ to be the space with the basis indexed by $[m+n]$ where $m$ and $n$ are lattice points in $K_{N}^{\vee}$ and $K_{N}$ respectively. It is naturally a module over $\mathbb{C}\left[K_{N}^{\vee} \oplus K_{N}\right]$.

Proposition 3.3.2. There is a natural isomorphism between the $B$ ring of Berglund-Hübsch construction, with components twisted by certain one-dimensional spaces, and the cohomology of

$$
\mathbb{C}\left[\left(K_{N}^{\vee} \oplus K_{N}\right)_{0}\right] \otimes \Lambda^{*}\left(N_{\mathbb{C}}\right)
$$

with respect to

$$
\begin{aligned}
d^{B} & :=\sum_{m \in \Delta}[m] \otimes(\text { contr. } m)+\sum_{n \in \Delta^{\vee}}[n] \otimes(\wedge n) \\
& =\sum_{m \in \Delta}[m] \otimes(\text { contr. } m)+\sum_{j=1}^{d}\left[v_{j}\right] \otimes\left(\wedge v_{j}\right)
\end{aligned}
$$

where we use the combinatorial reformulation of Section 2.

Proof. Denote by $N_{0}$ the lattice generated by $v_{j}, j=1, \ldots, n$, see Proposition 2.3.1. The complex under consideration splits into a direct sum of complexes that correspond to elements $N / N_{0}$, i.e. to elements of $G$.

Consider one element $g \in G$ and the corresponding rational linear combination $n_{g}=\sum_{j=1}^{d} h_{j} v_{j}$ with $0 \leq h_{j}<1$. The corresponding action on $x_{j}$ is $x_{j} \mapsto \exp \left(2 \pi \mathrm{i} h_{j}\right) x_{j}$. The corresponding part of the complex will have $n$ of the form $n_{g}+\sum_{j=1}^{d} \mathbb{Z}_{\geq 0} v_{j}$. The property $m \cdot n=0$ implies that only monomials in the variables $x_{j}$ that are fixed under $g$ can appear in the $g$-part of $\mathbb{C}\left[\left(K_{N}^{\vee} \oplus K_{N}\right)_{0}\right] \otimes \Lambda^{*}\left(N_{\mathbb{C}}\right)$.

The addition to $n$ of $v_{j}$ for which $h_{j}>0$ thus does not affect the $m$ that can occur. The $g$-part of $\mathbb{C}\left[\left(K_{N}^{\vee} \oplus K_{N}\right)_{0}\right] \otimes \Lambda^{*}\left(N_{\mathbb{C}}\right)$ is then seen to be the tensor product of two complexes. The first complex is given by

$$
\mathbb{C}\left[\sum_{j, h_{j}>0} \mathbb{Z}_{\geq 0} v_{j}\right] \otimes \Lambda^{*}\left(\oplus_{j, h_{j}>0} \mathbb{C} e_{j}\right)
$$

with the differential $\sum_{j, h_{j}>0}\left[v_{j}\right] \otimes\left(\wedge v_{j}\right)$. The second complex is precisely the complex of Proposition 3.2 .2 for the group $G$ and the variables $x_{j}$ that are fixed by $g$, with $\left[v_{j}\right]$ serving as $y_{j}$. These variables $x_{j}$ correspond to the elements of the dual basis to $\left(v_{j}\right)$.

The cohomology of the first complex is one-dimensional and is represented by $\mathbb{C} \Lambda_{j, h_{j}>0} v_{j}$. The cohomology of the second complex is given by Proposition 3.2 .2 as the $G$-equivariant part of $\mathcal{L}_{g}$. This gives the natural isomorphism claimed in the statement of the proposition. 
Remark 3.3.3. The arguments of Lemma 3.2.1 and Proposition 3.3.2 imply that the $g$-part of the $B$ ring in our combinatorial description comes from elements of the form

$$
\left[m \oplus n_{g}\right] \otimes \Lambda_{j, h_{j}>0} v_{j}
$$

where $n_{g}$ is the element that corresponds to $g$ and $m$ ranges over lattice elements in the interior of the face of $K_{N}^{\vee}$ with $m \cdot n_{g}=0$.

We will now show that the above isomorphism preserves the structures of the double graded supervector spaces. We will first describe the double grading and parity in our combinatorial formulation. We follow Definition 3.1.3 and define the conformal and cohomological grading of $(m \oplus n) \otimes P$ in $\mathbb{C}\left[\left(K_{N}^{\vee} \oplus K_{N}\right)_{0}\right] \otimes \Lambda^{*}\left(N_{\mathbb{C}}\right)$ by $m \cdot \operatorname{deg}{ }^{\vee}-\operatorname{deg} \cdot n+$ $\operatorname{deg}(P)-\operatorname{deg} \cdot \operatorname{deg}^{\vee}$ and $m \cdot \operatorname{deg}^{\vee}+\operatorname{deg} \cdot n-\operatorname{deg} \cdot \operatorname{deg}^{\vee}$ respectively. We also define parity as follows.

Proposition 3.3.4. The superspace structure on the cohomology of $\mathbb{C}\left[\left(K_{N}^{\vee} \oplus K_{N}\right)_{0}\right] \otimes \Lambda^{*}\left(N_{\mathbb{C}}\right)$ given by the sum of the cohomological and conformal grading modulo two is coming from the parity of the degree in $\Lambda^{*}\left(N_{\mathbb{C}}\right)$.

Proof. Clear.

Proposition 3.3.5. The double grading and parity on the $B$ ring given by conformal and cohomological gradings coincides with the grading on the $B$ ring given in [Kra].

Proof. The double grading on the $B$ ring was first given by Kaufmann, see [Kau1-[Kau3]. We will be using its description in [Kra]. Namely, the bi-grading on the $g$-component of the $B$ ring is

$$
\left(Q_{+}^{B}, Q_{-}^{B}\right)=\left(\sum_{h_{j} \neq 0}\left(h_{j}-q_{j}\right), \sum_{h_{j} \neq 0}\left(1-h_{j}-q_{j}\right)\right)+(p, p)
$$

where $p$ is the degree of the polynomial in the Milnor ring. Here $n_{g}=$ $\sum_{j=1}^{d} h_{j} v_{j}$ as in the proof of Proposition 3.3.2.

The element in (5) corresponds to the element of the Milnor ring of degree equal to $m \cdot \operatorname{deg}^{\vee}-\sum_{h_{j}=0} q_{j}$, because we need to adjust for the product of the variables. As a result, its bi-grading according to Kra] is given by

$$
\begin{aligned}
& \left(\sum_{h_{j} \neq 0}\left(h_{j}-q_{j}\right)+\left(m \cdot \operatorname{deg}^{\vee}-\sum_{h_{j}=0} q_{j}\right), \sum_{h_{j} \neq 0}\left(1-h_{j}-q_{j}\right)+\left(m \cdot \operatorname{deg}^{\vee}-\sum_{h_{j}=0} q_{j}\right)\right) \\
& =\left(\sum_{h_{j} \neq 0} h_{j}+m \cdot \operatorname{deg}^{\vee}-\sum_{j=1}^{d} q_{j},-\sum_{h_{j} \neq 0} h_{j}+\sum_{h_{j} \neq 0} 1+m \cdot \operatorname{deg}^{\vee}-\sum_{j=1}^{d} q_{j}\right)
\end{aligned}
$$


BERGLUND-HÜBSCH MIRROR SYMMETRY VIA VERTEX ALGEBRAS 17

$=\left(\operatorname{deg} \cdot n_{g}+m \cdot \operatorname{deg}{ }^{\vee}-\operatorname{deg} \cdot \operatorname{deg}{ }^{\vee},-\operatorname{deg} \cdot n_{g}+\sum_{h_{j} \neq 0} 1+m \cdot \operatorname{deg}{ }^{\vee}-\operatorname{deg} \cdot \operatorname{deg}^{\vee}\right)$

which equals the cohomological and conformal grading respectively.

We can make similar statements about the double grading of the $A$ ring, based on the comparison of the $A$ and $B$ graded spaces.

Proposition 3.3.6. The A ring of Berglund-Hübsch construction, with components twisted by certain one-dimensional spaces, is naturally isomorphic to the cohomology of

$$
\mathbb{C}\left[\left(K_{N}^{\vee} \oplus K_{N}\right)_{0}\right] \otimes \Lambda^{*}\left(M_{\mathbb{C}}\right)
$$

with respect to

$$
d^{A}:=\sum_{m \in \Delta}[m] \otimes(\wedge m)+\sum_{n \in \Delta^{\vee}}[n] \otimes(\text { contr. } n) .
$$

The bi-grading by $Q_{+}^{A}$ and $Q_{-}^{A}$ of $[\mathrm{Kra}$ comes from the cohomological and conformal grading on the complex respectively. These gradings assign to $[m \oplus n] \otimes P$ the degrees $m \cdot \operatorname{deg}^{\vee}+\operatorname{deg} \cdot n-\operatorname{deg} \cdot \operatorname{deg}^{\vee}$ and $m \cdot \operatorname{deg}^{\vee}-\operatorname{deg} \cdot n+\operatorname{deg}(P)-\operatorname{deg} \cdot \operatorname{deg}^{\vee}$ respectively.

Proof. It has been observed in [Kra] that $A$ and $B$ rings are isomorphic as vector spaces and their bi-gradings satisfy $Q_{+}^{A}=Q_{+}^{B}$ and $Q_{-}^{A}=$ $d-2 \mathrm{deg} \cdot \operatorname{deg}^{\vee}-Q_{-}^{B}$. The analogous statement for our construction is clear in view of the natural isomorphism of $\Lambda^{*}\left(M_{\mathbb{C}}\right)$ and $\Lambda^{*}\left(N_{\mathbb{C}}\right)$.

Remark 3.3.7. The aforementioned one-dimensional spaces are rather important. For example, they may easily switch the parity. From this point of view, the description of $A$ and $B$ rings of Berglund-Hübsch construction in terms of complexes, as opposed to Milnor rings, is more natural. Of course, there is a deeper meaning to these complexes which will become apparent in the vertex algebra setting.

\section{Vertex ALGEBra BACKGround}

In this section we will give an informal overview of vertex algebras, $N=2$ structures on vertex algebras, and the vertex algebras of $\sigma$ model type. In the latter setting we will define chiral rings. We will also describe the lattice vertex algebras constructed from a pair of dual lattices. All of the material can be found elsewhere, in particular in [Bo3] but is included here for the benefit of the reader. It provides the background necessary to understand Sections 5 and 6 which form the heart of the paper. 
4.1. Vertex algebras. A vertex algebra $V$ is a super vector space with an even element $|0\rangle$ called vacuum vector and a rather unusual structure $Y$ called state-field correspondence. This correspondence $Y$ : $V \rightarrow \operatorname{End}(V)\left[\left[z, z^{-1}\right]\right]$ assigns to every element $a \in V$ a power series

$$
Y(a, z)=a(z)=\sum_{l \in \mathbb{Z}} a_{(l)} z^{-l-1}
$$

in the variable $z$ where $a_{(l)}$ are endomorphisms of $V$. Power series $a(z)$ are called fields and $a_{(l)}$ are called their modes.

The correspondence $Y$ must satisfy a number of axioms, see $\mathrm{Kac}$. As a consequence of these axioms, every two fields $a(z)$ and $b(z)$ satisfy the operator product expansion (called OPE for short)

$$
a(z) b(w)=\sum_{j \leq r} \frac{c_{j}(w)}{(z-w)^{j}}
$$

where $r$ is some positive integer and $c_{j}(w)$ are other fields of the algebra. While all terms of the OPE are useful, one is especially interested in the poles along $z=w$. Consequently, the above OPE is often abbreviated as $a(z) b(w) \sim \sum_{j=1}^{r} \frac{c_{j}(w)}{(z-w)^{j}}$.

The so-called contour trick allows one to read off the supercommutators of the modes of $a(z)$ and $b(z)$ from the singular (i.e. $j>0$ ) part of the above OPE. Specifically, the series of supercommutators $\left[a_{(l)}, b(w)\right]$ is given in the above notations by

$$
\left[a_{(l)}, b(w)\right]=\operatorname{Res}_{z=w}\left(\sum_{j>0} \frac{z^{l} c_{j}(w)}{(z-w)^{j}}\right) .
$$

In particular, if the above OPE is nonsingular, i.e. $c_{j}=0$ for $j>0$, then all modes of $a$ and $b$ supercommute.

Remark 4.1.1. Vertex algebras are sometimes called chiral algebras, since only holomorphic (a.k.a. chiral) variable $z$ is used, as opposed to $z$ and $\bar{z}$.

4.2. Vertex algebras of $\sigma$-model type and chiral rings. Vertex algebras often come equipped with additional structures. The most common additional structure is that of a representation of the Virasoro algebra. This means a choice of an even field $L(z)$ of $V$ with a certain OPE with itself that translates into the following commutator relations for the modes $L[k]:=L_{(k+1)}=\operatorname{Res}_{z=0} L(z) z^{k+1}$

$$
[L[k], L[l]]=(k-l) L[k+l]+\frac{c}{12} \delta_{k+l}^{0}\left(k^{3}-k\right)
$$


where $\delta$ is the Kronecker symbol. These are the relations of the Virasoro algebra with central charge $c$. In addition, $L[0]$ is assumed to provide a grading on $V$ and $L[1]$ is assumed to correspond to differentiation of the fields, see [Kac]. The $N=2$ structure extends the Virasoro algebra to a somewhat larger superalgebra. It is characterized by a choice of three more fields $J(z), G^{+}(z)$ and $G^{-}(z)$, in addition to $L(z)$, which satisfy certain OPEs, see for example Bo3]. It appears naturally in the study of $\sigma$-models with Calabi-Yau target manifolds. Traditionally, the central charge $c$ is wriiten as $3 \hat{c}$ since $\hat{c}$ corresponds to the dimension of the Calabi-Yau. The algebras with such $N=2$ structures are called $N=2$ vertex algebras of central charge $\hat{c}$.

The following definition lies at the heart of mirror symmetry as it was originally understood by physicists.

Definition 4.2.1. For any vertex algebra with an $N=2$ structure, another such structure can be constructed by switching $G^{+}$with $G^{-}$, sending $J$ to $-J$ and keeping $L$ unchanged. This involution on the set of $N=2$ structures is called the mirror involution.

The operators $L[0]=L_{(1)}$ and $J_{(0)}$ are of special interest. They commute with each other and typically provide a double grading on $V$ (although this grading is not to be confused with the double grading on the $A$ and $B$ rings that was considered in the previous section). The following definition was introduced in Bo3] to codify other desirable properties of the $N=2$ vertex algebras.

Definition 4.2.2. We call an $N=2$ vertex algebra $V$ an $N=2$ vertex algebra of $\sigma$-model type if the eigenspaces $V_{\alpha}$ of $L_{(1)}$ are finitedimensional for all $\alpha$ and are zero except for $\alpha \in \frac{1}{2} \mathbb{Z}_{\geq 0}$, and the operators $H_{A}:=L_{(-1)}-\frac{1}{2} J_{(0)}$ and $H_{B}:=L_{(1)}+\frac{1}{2} J_{(0)}$ have only nonnegative integer eigenvalues.

Definition 4.2.3. The zero eigenspaces of $H_{A}$ and $H_{B}$ of $N=2$ algebra $V$ of $\sigma$-model type are called the $A$ and $B$ chiral rings of $V$ respectively.

The following proposition can be found in [LeVW]. While the paper overall has a number of string theory arguments, the proof of this proposition is completely mathematical.

Proposition 4.2.4. Let $v_{1}, v_{2}$ be elements of the $A$ chiral ring of $V$ (or both are in the $B$ chiral ring of $V$ ). Then their $O P E$ is nonsingular

$$
v_{1}(z) v_{2}(w)=v_{3}(w)+\sum_{j<0} \frac{c_{j}(w)}{(z-w)^{j}} .
$$


Moreover, $v_{1} \bullet v_{2}:=v_{3}$ defines a supercommutative product on the $A$ chiral ring (respectively $B$ chiral ring).

Proof. The idea of the proof is that if the OPE of $v_{1}$ and $v_{2}$ had a singular part, then this singular part would have elements with $H_{A}<0$ (resp. $\left.H_{B}<0\right)$. Since this is not possible, the OPE is nonsingular. Then the product properties follow from general properties of OPEs.

Remark 4.2.5. Chiral rings of $N=2$ vertex algebras of $\sigma$-model type are naturally equipped with conformal grading that comes from $2 L_{(1)}=$ $\pm J_{(0)}$. Vertex algebras $V$ that occur in this paper have an additional finite cohomological grading. This grading should be thought of as some rudimental manifestation of the antiholomorphic variables. As a result, the $A$ and $B$ rings of our theory come with a double grading.

Remark 4.2.6. The paper [LeVW] claims the existence of the so-called spectral flow, which in particular (at the parameter value 1) gives an isomorphism between $A$ and $B$ chiral rings of $V$ as vector spaces. It comes roughly speaking from $\mathrm{e}^{\int J(z)}$, if such field can be constructed. We will not attempt to axiomatize the spectral flow, but will rather construct it ad hoc for the vertex algebras under consideration.

4.3. Lattice vertex algebras. We will collect here the standard facts about the lattice vertex algebras which form the computational basis of our construction. Specifically, for a pair of dual lattices $M$ and $N$ we want to describe the vertex algebra Fock $_{M \oplus N}$. First, it contains a vertex subalgebra Fock $_{\mathbf{0} \oplus \mathbf{0}}$ which is generated by even (bosonic) fields $m^{\text {bos }}(z), n^{\text {bos }}(z)$ with OPEs

$$
m^{b o s}(z) m^{b o s}(w) \sim n^{b o s}(z) n^{b o s}(w) \sim 0, m^{b o s}(z) n^{b o s}(w) \sim \frac{m \cdot n}{(z-w)^{2}}
$$

and odd (fermionic) fields $m^{\text {ferm }}(z), n^{\text {ferm }}(z)$ with OPEs

$$
\begin{gathered}
m^{\text {ferm }}(z) m^{\text {ferm }}(w) \sim n^{\text {ferm }}(z) n^{\text {ferm }}(w) \sim 0, \\
m^{\text {ferm }}(z) n^{\text {ferm }}(w) \sim \frac{m \cdot n}{z-w} .
\end{gathered}
$$

The algebra Fock $_{\mathbf{0} \oplus \mathbf{0}}$ can be thought of as a polynomial ring in infinitely many even variables $\left(m_{i}\right)_{(<0)}^{\text {bos }},\left(n_{i}\right)_{(<0)}^{\text {bos }}$ and infinitely many odd variables $\left(m_{i}\right)_{(<0)}^{\text {ferm }},\left(n_{i}\right)_{(<0)}^{\text {ferm }}$ where $m_{i}$ and $n_{i}$ form bases of $M$ and $N$.

Note that the endomorphisms $m_{(0)}^{\text {bos }}$ and $n_{(0)}^{\text {bos }}$ act by zero on Fock $\mathbf{0}_{\mathbf{0}} \mathbf{0}$. As we pass to Fock $_{M \oplus N}$, we introduce other eigenspaces of these operators. The algebra Fock $_{M \oplus N}$ is isomorphic as a vector space to Fock $_{\mathbf{0} \oplus \mathbf{0}} \otimes_{\mathbb{C}} \mathbb{C}[M \oplus N]$. There are additional operators $\mathrm{e}^{\int m^{\text {bos }}(z)+n^{\text {bos }}(z)}$ 
whose construction is rather subtle (but is standard in the field of vertex algebras). One needs to use the normal ordering, as well as some explicit cocycle. The reader is referred to [Bo3] for more details.

The OPEs of $\mathrm{e}^{\int m^{b o s}(z)+n^{b o s}(z)}$ with fermionic generators are nonsingular. The OPEs of $\mathrm{e}^{\int m^{\text {bos }}(z)+n^{\text {bos }}(z)}$ with $\tilde{m}^{\text {bos }}(z)$ and $\tilde{n}^{\text {bos }}(z)$ are

$$
\begin{aligned}
& \tilde{m}^{b o s}(z) \mathrm{e}^{\int m^{b o s}(w)+n^{b o s}(w)}=\frac{\tilde{m} \cdot n}{(z-w)} \mathrm{e}^{\int m^{b o s}(w)+n^{b o s}(w)} \\
& \tilde{n}^{b o s}(z) \mathrm{e}^{\int m^{b o s}(w)+n^{b o s}(w)}=\frac{m \cdot \tilde{n}}{(z-w)} \mathrm{e}^{\int m^{b o s}(w)+n^{b o s}(w)} .
\end{aligned}
$$

The OPEs of $\mathrm{e}^{\int m^{b o s}(z)+n^{b o s}(z)}$ and $\mathrm{e}^{\int \tilde{m}^{b o s}(z)+\tilde{n}^{b o s}(z)}$ are obtained from expanding

$\mathrm{e}^{\int m^{b o s}(z)+n^{b o s}(z)} \mathrm{e}^{\int \tilde{m}^{b o s}(z)+\tilde{n}^{b o s}(w)}=\mathrm{e}^{\int m^{b o s}(z)+\tilde{m}^{b o s}(w)+n^{b o s}(z)+\tilde{n}^{b o s}(w)}(z-w)^{m \cdot \tilde{n}+\tilde{m} \cdot n}$

in powers of $z-w$. In particular, the OPE is nonsingular iff the pairing $m \cdot \tilde{n}+\tilde{m} \cdot n$ is nonnegative. This fact will be used extensively throughout the calculations of the paper.

Remark 4.3.1. A version of Pauli exclusion principle implies that the OPE of $n^{\text {ferm }}$ with itself looks like

$$
n^{f e r m}(z) n^{f e r m}(w)=n^{f e r m}(w) \partial_{w} n^{f e r m}(w)(z-w)+O(z-w)^{2}
$$

because $n^{\text {ferm }}(w) n^{\text {ferm }}(w)=0$.

\section{Vertex Algebras of Berglund-HüBSCh MirRoR SYMMETRY: DEFINITIONS AND FIRST PROPERTIES}

In this section, we define the vertex algebras of Berglund-Hübsch mirror symmetry and prove their first properties. In particular, we extend the Key Lemma of [Bo2] to the Berglund-Hübsch setting, see Theorem 5.2.3.

\subsection{Definition of vertex algebras of Berglund-Hübsch mir-} ror symmetry. Let $W$ and $G$ be the Berglund-Hübsch potential and group, and let $M, N, K_{M}, K_{N}$, deg, $\operatorname{deg}^{\vee}, \Delta, \Delta^{\vee}$ be defined as in Subsection 2.3. Denote by $W^{\vee}$ and $G^{\vee}$ the dual potential and the dual group. 
Consider the lattice vertex algebra Fock $_{M \oplus N}$ with the $N=2$ structure

$$
\begin{aligned}
G^{+}(z)= & \sum_{i}\left(n^{i}\right)^{\text {bos }}(z)\left(m^{i}\right)^{\text {ferm }}(z)-\partial_{z} \operatorname{deg}^{\text {ferm }}(z) \\
G^{-}(z)= & \sum_{i}\left(m^{i}\right)^{\text {bos }}(z)\left(n^{i}\right)^{\text {ferm }}(z)-\partial_{z}\left(\operatorname{deg}^{\vee}\right)^{\text {ferm }}(z) \\
J(z)= & \sum_{i}\left(m^{i}\right)^{\text {ferm }}(z)\left(n^{i}\right)^{\text {ferm }}(z)+\operatorname{deg}^{\text {bes }}(z)-\left(\mathrm{deg}^{\vee}\right)^{\text {bos }}(z) \\
L(z)= & \sum_{i}\left(m^{i}\right)^{\text {bos }}(z)\left(n^{i}\right)^{\text {bos }}(z)+\frac{1}{2} \sum_{i} \partial_{z}\left(m^{i}\right)^{\text {ferm }}(z)\left(n^{i}\right)^{\text {ferm }}(z) \\
& -\frac{1}{2} \sum_{i} \partial_{z}\left(n^{i}\right)^{\text {ferm }}(z)\left(m^{i}\right)^{\text {ferm }}(z) \\
& -\frac{1}{2} \partial_{z} \operatorname{deg}^{\text {bos }}(z)-\frac{1}{2} \partial_{z}\left(\operatorname{deg}^{\vee}\right)^{\text {bos }}(z)
\end{aligned}
$$

with the normal ordering implicitly used in the definition of $J$ and $L$. This $N=2$ structure has central charge $\hat{c}=d-2 \mathrm{deg} \cdot \operatorname{deg}^{\vee}$. Consider the differential

$$
D_{\mathbf{1}, \mathbf{1}}:=\operatorname{Res}_{z=0}\left(\sum_{m \in \Delta} m^{f e r m}(z) \mathrm{e}^{\int m^{\text {bos }}(z)}+\sum_{n \in \Delta^{\vee}} n^{f e r m}(z) \mathrm{e}^{\int n^{b o s}(z)}\right)
$$

or more generally the differential

$$
D_{f, g}:=\operatorname{Res}_{z=0}\left(\sum_{m \in \Delta} f(m) m^{f e r m}(z) \mathrm{e}^{\int m^{b o s}(z)}+\sum_{n \in \Delta^{\vee}} g(n) n^{f e r m}(z) \mathrm{e}^{\int n^{b o s}(z)}\right)
$$

where $f$ and $g$ are generic coefficient functions.

Definition 5.1.1. The vertex algebra $V_{f, g}$ is defined as the cohomology of Fock $_{M \oplus N}$ with respect to the differential $D_{f, g}$.

Remark 5.1.2. To check that $D_{f, g}$ is indeed a differential, observe that its summands supercommute. This calculation is based on the property $m \cdot n \geq 0$ for all $m \in \Delta, n \in \Delta^{\vee}$. For exampe, if $m \cdot n=0$ then the OPE of $m^{\text {ferm }}(z) \mathrm{e}^{\int m^{\text {bos }}(z)}$ and $n^{\text {ferm }}(w) \mathrm{e}^{\int n^{\text {bos }}(w)}$ has no poles at $z=w$ coming from the bosons or from fermions. If $m \cdot n>0$ then this OPE has a pole of order one coming from the fermions, but a zero of order at least one coming from the bosons, so overall the OPE is nonsingular.

Proposition 5.1.3. The vertex algebra $V_{f, g}$ inherits the $N=2$ structure above from Fock $_{M \oplus N}$.

Proof. One explicitly calculates that $D_{f, g}$ supercommutes with $G^{ \pm}(z)$. It is important for this calculation that $m \cdot \operatorname{deg}^{\vee}=\operatorname{deg} \cdot n=1$ for all $m \in \Delta$ and $n \in \Delta^{\vee}$. Since $J$ and $L$ can be calculated from the supercommutators of $G^{ \pm}$, the statement follows.

Remark 5.1.4. Note that when we interchange $(W, G)$ with $\left(W^{\vee}, G^{\vee}\right)$, we simply interchange $M$ and $N$ and data therein. The corresponding algebra $V$ is then the same, but the $N=2$ structure differs by mirror involution that interchanges $G^{+}$and $G^{-}$, preserves $L$ and sends $J$ to 
$-J$. There is a minor subtlety related to the cocycle in the definition of the vertex operators, see [Bo2] for details, which are still applicable in the Berglund-Hübsch setting.

Remark 5.1.5. One can view $\operatorname{Fock}_{M \oplus N}, D_{f, g}$ as a complex, with the grading provided by $\operatorname{Fock}_{m \oplus n} \mapsto m \cdot \operatorname{deg}^{\vee}+\operatorname{deg} \cdot n$. We refer to the induced grading on $V_{f, g}$ as cohomological. We will later see that it is related to the cohomological grading on the $A$ and $B$ rings of BerglundHübsch construction.

Remark 5.1.6. The explicit formulas for the grading by $L_{(1)}$ and $J_{(0)}$ are given in [Bo3]. They can be briefly described as follows. The $\left(L_{(1)}, J_{(0)}\right)$ grading of $m^{\text {ferm }}$ and $n^{\text {ferm }}$ is $\left(\frac{1}{2}, 1\right)$ and $\left(\frac{1}{2},-1\right)$, the grading of $m^{\text {bos }}$ and $n^{\text {bos }}$ is $(1,0)$. Differentiation changes the grading by $(1,0)$. The vertex operator $\mathrm{e}^{\int m^{b o s}(z)+n^{b o s}(z)}$ has grading

$$
\left(m \cdot n+\frac{1}{2} m \cdot \operatorname{deg}^{\vee}+\frac{1}{2} \operatorname{deg} \cdot n,-m \cdot \operatorname{deg}^{\vee}+\operatorname{deg} \cdot n\right) .
$$

5.2. Key Lemma. In this subsection we will prove the BerglundHübsch analog of the Key Lemma of [Bo2]. We shall first formulate a commutative algebra result that will be used in the vertex algebra argument. Recall that we have dual lattices $M$ and $N$, the cones $K_{M}$ and $K_{N}$ with $K_{M} \cdot K_{N} \geq 0$ and the sets $\Delta$ and $\Delta^{\vee}$ in $M$ and $N$, which encode the monomials of $W$ and $W^{\vee}$ respectively. We will assume throughout that the coefficient functions $f$ and $g$ are generic, which in Berglund-Hübsch setting is equivalent to nonvanishing of all $f(m)$ and $g(n)$.

Proposition 5.2.1. For every one-dimensional face of $K_{N}^{\vee}$ there exists a nonzero lattice point $v$ on it and elements $P_{n} \in \mathbb{C}[M]$ such that

$$
[v]=\sum_{n \in \Delta^{\vee}}\left(P_{n} \sum_{m \in \Delta} f(m)(m \cdot n)[m]\right)
$$

where $P_{n}$ have the additional property that all of their monomials $[w]$ satisfy $w \cdot n \geq-1$ and $w \cdot \hat{n} \geq 0$ for all $\hat{n} \in \Delta^{\vee}$ for $\hat{n} \neq n$.

Proof. Recall that $\mathbb{C}\left[K_{N}^{\vee}\right]$ is the $G$-invariant subring of the polynomial ring $\mathbb{C}\left[x_{1}, \ldots, x_{d}\right]$, see Section 2. The monomials in the potential $W$ correspond to the elements $m \in \Delta$. The rays of $K_{N}^{\vee}$ correspond to variables $x_{i}$. Without loss of generality, we may assume that the ray in question corresponds to $x_{1}$. Then the monomials $[v]$ correspond to monomials $x_{1}^{l}$. Here $l$ is a multiple of some number, determined by the condition that this monomial is $G$-invariant. 
Since the potential $W$ is nondegenerate, the partial derivatives $\partial_{i} W$ form a regular sequence in $\mathbb{C}\left[x_{1}, \ldots, x_{d}\right]$. As a result, the quotient by these elements is finite-dimensional. It is also graded (we assign $x_{i}$ some positive weights $q_{i}$ ). Thus for all sufficiently large $l$ the monomial $x_{1}^{l}$ lies in the ideal generated by $\partial_{i} W, i=1, \ldots, d$. Pick one such $l$ with the additional property that $x_{1}^{l}$ is $G$-invariant. There now exist polynomials $\tilde{P}_{i}(x)$ such that

$$
x_{1}^{l}=\sum_{i=1} \tilde{P}_{i}(x) \partial_{i} W
$$

which we can rewrite as

$$
x_{1}^{l}=\sum_{i=1} P_{i}(x)\left(x_{i} \partial_{i} W\right)
$$

with $P_{i}(x)=\frac{\tilde{P}_{i}(x)}{x_{i}}$. Observe now that $x_{i} \partial_{i} W$ is a $G$-invariant polynomial given by $\sum_{m \in \Delta} f(m)\left(m \cdot n_{i}\right)[m]$ for the corresponding element $n_{i} \in \Delta^{\vee}$. We can drop all of the monomials in $P_{i}(x)$ that are not invariant under the group $G$. We keep calling the resulting polynomials $P_{i}(x)$ and observe that they correspond to $P_{i} \in \mathbb{C}[M]$ that satisfy the conditions of the proposition.

Corollary 5.2.2. For every facet of $\theta \subset K_{N}$ there exist $\hat{P}_{n} \in \mathbb{C}[M]$ such that

$$
[0]=\sum_{n \in \Delta^{\vee}}\left(\hat{P}_{n} \sum_{m \in \Delta} f(m)(m \cdot n)[m]\right)
$$

where $\hat{P}_{n}$ have the following additional property. For any $n \in \Delta$ and any $\hat{n} \in \theta$ all monomials $[w]$ of $P_{n}$ satisfy $w \cdot \hat{n} \geq-1$ if $\hat{n}=n$ and $w \cdot \hat{n} \geq 0$ if $\hat{n} \neq n$.

Proof. We simply divide both sides of the result of Proposition 5.2.1 by $[v]$ that lies on the ray of $K_{N}^{\vee}$ which is dual to $\theta$.

The Key Lemma below reduces the cohomology of Fock ${ }_{M \oplus N}$ by $D_{f, g}$ to the cohomology of Fock $K \oplus N$ or Fock $M \oplus K_{N}$. The idea is the following. The operator $D_{f, g}$ is the sum of two parts, one coming from $\Delta$ and the other coming from $\Delta^{v}$. These form two differentials of the double complex, if $\operatorname{Fock}_{M \oplus N}$ is graded by assigning $\left(m \cdot \operatorname{deg}^{\vee}\right.$, deg $\left.\cdot n\right)$ to Fock $_{(m, n)} \subseteq$ Fock $_{M \oplus N}$. However, the stupid filtrations do not converge. In fact, the cohomology of the total complex is nontrivial, while the cohomology with respect to the horizontal or the vertical part of the differential are both trivial. We will find various homotopies to identity for one of the differentials, such that their anticommutator with the other will have certain properties. 
Theorem 5.2.3. Let $f$ be a generic coefficient function. Then the cohomology of Fock $_{M \oplus N}$ with respect to $D_{f, g}$ is equal to the cohomology of Fock $_{M \oplus K_{N}}$ with respect to $D_{f, g}$.

Proof. We follow the method of Propositions 8.1 and 8.2 of [Bo2]. Let $m_{0}$ be a generator of a ray of $K_{N}^{\vee}$ and $\theta$ the corresponding facet of $K_{N}$ Consider the operator on Fock $_{M \oplus N}$ given by

$$
R_{\theta}:=\operatorname{Res}_{z=0} \sum_{n \in \Delta^{\vee}} n^{\text {ferm }}(z) \hat{P}_{n}(z)
$$

where $\hat{P}_{n}$ is given by Corollary 5.2 .2 in the sense that every every monomial $[p]$ of $\hat{P}_{n}$ is converted into $\mathrm{e}^{\int p^{b o s}(z)}$.

It is a standard calculation to show that for any $v \in$ Fock $_{M \oplus N}$

$$
R_{\theta} D_{f, g} v+D_{f, g} R_{\theta} v=v+\alpha_{\theta} v
$$

where $\alpha_{\theta}$ increases $m_{0} \bullet$ and does not decrease $\hat{m}_{0} \bullet$ for other generators of rays of $K_{N}^{\vee}$. Indeed, the definition of $\hat{P}_{n}$ implies the OPE of

$$
\sum_{n \in \Delta^{\vee}} n^{f e r m}(z) \hat{P}_{n}(z)\left(\sum_{m \in \Delta} f(m) m^{f e r m}(w) \mathrm{e}^{\int m^{b o s}(w)}\right) \sim \frac{1}{z-w}
$$

which in turn implies that the anticommutator of $R_{\theta}$ with the part of $D_{f, g}$ that comes from $M$ is the identity. As a result, $\alpha_{\theta}$ is the anticommutator of $R_{\theta}$ with the $N$-part of $D_{f, g}$. Since $n \in \Delta^{\vee}$ pair up nonnegatively with all $\hat{m}_{0}$, it remains to show that for $\hat{n} \in \theta$ the corresponding anticommutator is zero. It then suffices to show that the OPE

$$
\sum_{n \in \Delta \vee} n^{f e r m}(z) \hat{P}_{n}(z) \hat{n}^{f e r m}(w) \mathrm{e}^{\int \hat{n}^{b o s}(w)}
$$

is nonsingular. This is assured by the conditions on monomials of $P_{n}$. If $n \neq \hat{n}$, then the OPEs of all of the ingredients above are nonsingular. If $n=\hat{n}$, then the OPE of the bosonic parts may potentially have a pole of order one, along $z=w$ but it is counteracted by zero of order one coming from $n^{\text {ferm }}(z) n^{\text {ferm }}(w)$, see Remark 4.3.1,

The argument of Proposition 8.2 of [Bo2] now finishes the proof.

Remark 5.2.4. The difference between Berglund-Hübsch and BatyrevBorisov settings is that in Berglund-Hübsch case we have to worry about the $n=\hat{n}$ in Corollary 5.2.2. It corresponds to the difference between a potential that is nondegenerate in the sense of Batyrev [Ba1, i.e. in the logarithmic coordinates, and the potential that is nondegenerate in the sense of Berglund-Hübsch, i.e. in the usual coordinates. 
5.3. Fields of the spectral flow. In this subsection we will provide two examples of fields of $V_{f, g}$ that will be useful later. They should be thought of as $\mathrm{e}^{\int \pm J(z)}$. Consider the fields of Fock $_{M \oplus N}$ given by

$$
\mathrm{SF}_{+}(z)=\mathrm{e}^{\int \operatorname{deg}^{\text {bos }}(z)-\left(\operatorname{deg}^{\vee}\right)^{\text {bos }}(z)} \Lambda^{d} M^{f e r m}(z)
$$

and

$$
\mathrm{SF}_{-}(z)=\mathrm{e}^{\int-\operatorname{deg}^{b o s}(z)+\left(\operatorname{deg}^{\vee}\right)^{b o s}(z)} \Lambda^{d} N^{f e r m}(z) .
$$

A specific choice of the generator of $\Lambda^{d} N^{f e r m}(z)$ is not easy to fix, but we can use an integral basis of $N$ to do so up to \pm 1 .

Proposition 5.3.1. Fields $\mathrm{SF}_{ \pm}(z)$ descend to fields of $V_{f, g}$. They have cohomological degree zero. They have $L_{(1)}$ degree $\frac{\hat{c}}{2}$ and $J_{(0)}$ degree $\pm \hat{c}$. In particular, $\mathrm{SF}_{+}$lies in the $A$ chiral ring of $V_{f, g}$ and $\mathrm{SF}_{-}$lies in its $B$ chiral ring.

Proof. Note that the OPEs of $\mathrm{SF}_{ \pm}$with the fields $m^{\text {ferm }}(z) \mathrm{e}^{\int m^{\text {bos }}(z)}, m \in$ $\Delta$ and $n^{\text {ferm }}(z) \mathrm{e}^{\int n^{\text {bos }}(z)}, n \in \Delta^{\vee}$ are nonsingular. Indeed, the poles of order one at $z=w$ for the bosonic part are counteracted by the zeroes of order one for the fermionic part and vice versa. Thus $\mathrm{SF}_{ \pm}(z)$ supercommute with $D_{f, g}$ and descend to fields of $V_{f, g}$. Their modes then descend to endomorphisms of $V_{f, g}$. The degree statements are consequences of the Remark 5.1.6.

\section{Main TheOrem}

In this section we will use the vertex algebra machinery to provide a natural isomorphism between the $B$ ring of the Berglund-Hübsch pair $(W, G)$ and the $A$ ring of dual pair $\left(W^{\vee}, G^{\vee}\right)$. We are working in the notations of the previous section.

6.1. Four subcomplexes. Recall that the vertex algebra $V_{f, g}$ is the cohomology of Fock $M \oplus N$ with respect to $D_{f, g}$. We will describe four subcomplexes of Fock $_{M \oplus N}$ which are naturally isomorphic to the complexes of Section 3 when $(f, g)=(\mathbf{1}, \mathbf{1})$.

Let us denote by $\mathcal{C}_{K_{N}^{\vee}-\operatorname{deg}, K_{N}, N}$ the subspace of Fock ${ }_{M \oplus N}$ spanned by the elements that corresponds to the fields of the form

$$
\mathrm{e}^{\int m^{b o s}(z)-\operatorname{deg}^{b o s}(z)+n^{b o s}(z)} P\left(\tilde{n}^{f e r m}(z)\right)
$$

where $m \in K_{M}, n \in K_{M}^{\vee}$ with $m \cdot n=0$ and $P$ is some polynomial in the odd anticommuting fields $\tilde{n}^{\text {ferm }}(z), \tilde{n} \in N$. Three more subspaces $\mathcal{C}_{K_{M}-\operatorname{deg}, K_{M}^{\vee}, N}, \mathcal{C}_{K_{N}^{\vee}, K_{N}-\operatorname{deg}^{\vee}, M}$ and $\mathcal{C}_{K_{M}, K_{M}^{\vee}-\operatorname{deg}^{\vee}, M}$ are defined similarly. 
Proposition 6.1.1. The four subspaces

$$
\mathcal{C}_{K_{N}^{\vee}-\operatorname{deg}, K_{N}, N}, \mathcal{C}_{K_{M}-\operatorname{deg}, K_{M}^{\vee}, N}, \mathcal{C}_{K_{N}^{\vee}, K_{N}-\operatorname{deg} \vee, M} \mathcal{C}_{K_{M}, K_{M}^{\vee}-\operatorname{deg} \vee, M}
$$

of Fock $_{M \oplus N}$ are preserved under the action of $D_{f, g}$. For $(f, g)=(\mathbf{1}, \mathbf{1})$, the $D_{f, g}$ cohomology of these complexes is naturally isomorphic to the $B$ ring of $(W, G)$, A ring of $\left(W^{\vee}, G^{\vee}\right), A$ ring of $(W, G)$ and $B$ ring of $\left(W^{\vee}, G^{\vee}\right)$ respectively.

Proof. Let us first focus on $\mathcal{C}_{K_{N}^{\vee}-\operatorname{deg}, K_{N}, N}$. The action of $D_{f, g}$ is computed by looking at the OPEs. The OPE of (17) with $m_{1}^{\text {ferm }}(w) \mathrm{e}^{\int m_{1}^{\text {bos }}(w)}$ for $m_{1} \in \Delta$ has a pole of order one at $z=w$ coming from the fermionic terms, with the residue given by the contraction of $P$ by $m_{1}$. The action of the corresponding term of $D_{f, g}$ can thus be nonzero only if the bosonic terms introduce no positive powers of $(z-w)$, i.e. $m_{1} \cdot n=0$ (recall that $n \in K_{N} \subseteq K_{M}^{\vee}$, so $m_{1} \cdot n \geq 0$ ). Similarly, the OPE of (17) with $n_{1}^{\text {ferm }}(w) \mathrm{e}^{\int n_{1}^{\text {bos }}(w)}$ for $n_{1} \in \Delta$, has no poles coming from the fermionic parts and the pole of order at most one coming from the bosons. The pole of order one is realized when $(m-\operatorname{deg}) \cdot n_{1}=-1$ which means $m \cdot n_{1}=0$. It is then easy to see that for $(f, g)=(\mathbf{1}, \mathbf{1})$ the complex is naturally isomorphic to that of Proposition 3.3.2.

The statement about the $B$ ring of $\left(W^{\vee}, G^{\vee}\right)$ is obtained by switching $M$ and $N$. The statements about the $A$ rings follow similarly, by an OPE calculation and Proposition 3.3.6.

Remark 6.1.2. While we are guaranteed that the cohomology spaces of the above four subcomplexes map to $V_{f, g}$, it is not at all obvious that these maps are injective. It will turn out to be the case, moreover the images of the cohomology spaces of the first and second subcomplexes coincide with the $B$ ring of $V_{f, g}$ and the images of the cohomology spaces of the third and fourth subcomplexes coincide with the $A$ ring of $V_{f, g}$, see the proof of Theorem 6.2.1 below.

6.2. Main theorem. We are now ready to formulate and prove the main theorem of this paper. Let $W$ and $G$ be the Berglund-Hübsch potential and the group, and let $M, N, K_{M}, K_{N}, \operatorname{deg}, \operatorname{deg}^{\vee}, \Delta, \Delta^{\vee}$ be defined as in Subsection 2.3. Denote by $W^{\vee}$ and $G^{\vee}$ the dual potential and the dual group.

Theorem 6.2.1. For generic choices of $f$ and $g$ the $N=2$ vertex algebra $D_{f, g}$ is of $\sigma$-model type. In particular, this algebra is of $\sigma$ model type for $(f, g)=(\mathbf{1}, \mathbf{1})$. The $B$ ring of $V_{\mathbf{1}, \mathbf{1}}$ can be identified with the $B$ ring of $(W, G)$ and with the $A$ ring of $\left(W^{\vee}, G^{\vee}\right)$. The $A$ ring of $V_{\mathbf{1}, \mathbf{1}}$ can be identified with the $A$ ring of $(W, G)$ and with the $B$ ring of $\left(W^{\vee}, G^{\vee}\right)$. 
Proof. We will first show that the $B$ and the $A$ rings of $V_{f, g}$ are isomorphic to the cohomology spaces of the first and the fourth subcomplexes of Proposition 6.1.1, respectively.

Theorem 5.2.3 implies that the vertex algebra $V_{f, g}$ is the cohomology of Fock $_{K_{M} \oplus N}$ by $D_{f, g}$. We will write $D_{f, g}=D_{f}+D_{g}$ where $D_{f}$ denotes the terms that come from $M$ and $D_{g}$ denotes the terms that come from $N$. We now apply Proposition 6.3 of [Bo3]. It asserts that for strongly nondegenerate coefficient functions $f$ the $D_{f}$-cohomology of Fock $_{K_{M} \oplus N}$ has $H_{A} \geq 0$ and moreover the $H_{A}=0$ part (i.e. the $A$ chiral ring of

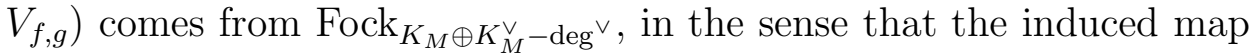
on the $H_{A}=0$ graded pieces of cohomology is an isomorphism. While Proposition 6.3 of [Bo3] was stated in the setting of Batyrev-Borisov mirror symmetry, it does not use full combinatorial ingredients of the construction and so it applies to Berglund-Hübsch case.

Generic $f$ are strongly nondegenerate (the definition of strong nondegeneracy is given in [Bo3]). Because of the scaling symmetries, all $f$ with $f(m) \neq 0, \forall m \in \Delta$, and in particular $f=\mathbf{1}$, have the same behavior and are thus strongly nondegenerate.

Remark 6.2.2. It is worth mentioning that the proof of Proposition 6.3 of [Bo3] is in fact rather easy in the Berglund-Hübsch case, since one does not need to do any fan subdivisions of $K_{M}$. However, we chose to quote Bo3 since the full power of the argument will be necessary when we unify the two constructions in the next section.

Now, as in Proposition 7.3 of [Bo3], we observe that the $H_{A}=0$ part of Fock $_{K_{M} \oplus K_{M}^{\vee}-\operatorname{deg} \vee}$ is made of linear combinations of elements that correspond to fields of the form

$$
\mathrm{e}^{\int m^{b o s}(z)+n^{\text {bos }}(z)-\left(\operatorname{deg}^{\vee}\right)^{b o s}(z)} P\left(\tilde{m}^{f e r m}(z)\right)
$$

where $m \in K_{M}, n \in K_{M}^{\vee}$ with $m \cdot n=0$. Thus it comes from the fourth complex of Proposition 6.1.1. By switching $M$ and $N$, we see that the $B$ ring of $V_{f, g}$ is calculated by the first complex of Proposition 6.1.1. Also, the results of [Bo3] ensure that for generic $(f, g)$ the vertex algebra $V_{f, g}$ is of $\sigma$-model type. The reader may be somewhat concerned about the proof of the statement that $L_{(1)}$ graded components of $V_{f, g}$ are finitedimensional, since the argument of [Bo3] refers to [BoL]. However, one can simply use Proposition 6.5 of [Bo3] which states that a fixed $H_{A}$ eigenspace of $V_{f, g}$ comes from Fock $_{K_{M} \oplus K_{M}^{\vee}-r \text { deg }}$ for some $r$. If one first takes cohomology with respect to $D_{g}$, then we can reduce $m$ to a finite list of possibilities. Then it remains to observe that for a fixed $m$ and $r$ the bigraded component of Fock $_{m \oplus K_{M}^{\vee}-r \operatorname{deg}^{\vee}}$ is finite-dimensional. 
Indeed, in the formula of Remark 5.1.6 we have $m \cdot n \geq-r m \cdot \operatorname{deg}^{\vee}$, and thus deg $\cdot n$ is bonded from above. Thus, we have a finite list of possible $n$ as well, and then the fact that $L_{(1)}$ is fixed implies the finiteness.

We will now work to give alternative descriptions of $A$ and $B$ rings of $V_{f, g}$ by means of the third and second complexes of Proposition 6.1.1 respectively. We can no longer directly appeal to the results of [Bo3]. Instead, we will use the idea of the spectral flow of [LeVW].

An easy calculation of grading, see Remark 5.1.6, shows that the cohomology of the second complex maps inside the $B$ ring of $V_{f, g}$, and the cohomology of the third complex maps inside the $A$ ring of $V_{f, g}$. On the other hand, there are natural isomorphisms of complexes between the second and the fourth complexes, as well as the first and the third complexes, which we construct below.

Consider a $\left(L_{(1)}, J_{(0)}\right)$ degree $\left(\frac{k}{2},-k\right)$ element of the second complex. It is a linear combination of elements

$$
\mathrm{e}^{\int m^{b o s}(z)-\operatorname{deg}^{b o s}(z)+n^{b o s}(z)} P\left(\tilde{n}^{f e r m}\right), m \in K_{M}, n \in K_{M}^{\vee}
$$

with $m \cdot \operatorname{deg}^{\vee}-\operatorname{deg} \cdot \operatorname{deg}^{\vee}-\operatorname{deg} \cdot n+\operatorname{deg}(P)=k$. If we consider the OPE of the above element with the spectral flow operator $\mathrm{SF}_{+}$of Proposition 5.3 .1

$$
\mathrm{SF}_{+}(z)=\mathrm{e}^{\int \operatorname{deg}^{\text {bos }}(z)-\left(\operatorname{deg}^{\vee}\right)^{b o s}(z)} \Lambda^{d} M^{f e r m}(z)
$$

the leading term will be

$$
(z-w)^{-k} \mathrm{e}^{\int m^{b o s}(w)+n^{b o s}(w)-\left(\operatorname{deg}^{\vee}\right)^{b o s}(w)} P^{\vee}\left(\tilde{m}^{f e r m}\right), m \in K_{M}, n \in K_{M}^{\vee}
$$

where $P^{\vee}$ is obtained by contracting $\Lambda^{d} M_{\mathbb{C}}$ with $P$. This gives an element of the fourth complex with $\left(L_{(1)}, J_{(0)}\right)$ grading $\left(\frac{\hat{c}-k}{2}, \hat{c}-k\right)$. There is a natural map in the opposite direction given by reading off the leading term of $\mathrm{OPE}$ with $\mathrm{SF}_{-}$. Clearly, these two maps are inverses of each other in Fock $_{M \oplus N}$. Since $\mathrm{SF}_{ \pm}$are fields of $V_{f, g}$ and since reading off the leading term of the OPE makes sense in $V_{f, g}$, these two maps give inverses of each other as maps between the image of the cohomology of the second complex and the cohomology of the fourth complex (i.e. the $A$ ring of $\left.V_{f, g}\right)$.

We have thus constructed an injective map from the $A$ ring of $V_{f, g}$ to the $B$ ring of $V_{f, g}$ which sends an element of $\left(L_{(1)}, J_{(0)}\right)$ degree $\left(\frac{l}{2}, l\right)$ to an element of degree $\left(\frac{\hat{c}-l}{2}, l-\hat{c}\right)$ by reading off the $(z-w)^{-l}$ term of $\mathrm{OPE}$ with $\mathrm{SF}_{-}$. Similarly, there is an injective map in the other direction given by reading off the appropriate term of OPE with $\mathrm{SF}_{+}$. Since the dimensions of the $A$ and $B$ rings are finite, it means that they 
coincide. In addition, we see that the above spectral flow produces a vector space isomorphism of $A$ and $B$ rings.

It remains to observe that since $(\mathbf{1}, \mathbf{1})$ is a pair of strongly nondegenerate coordinate functions, the above argument works for it. Then Proposition 6.1.1 finishes the proof of Theorem 6.2.1.

Remark 6.2.3. Since the cohomological grading of $\mathrm{SF}_{ \pm}$is zero, the spectral flow isomorphism constructed in the proof of Theorem 6.2.1 preserves the cohomological grading. Also observe that the spectral flow isomorphism corresponds naturally to passing between $\Lambda^{*}(N)$ and $\Lambda^{*}(M)$.

Remark 6.2.4. It is important to point out that the isomorphism between the $B$ ring of $(W, G)$ and the $A$ ring of $\left(W^{\vee}, G^{\vee}\right)$ constructed in Theorem 6.2.1 is not easy to write explicitly. It means passing from fields of the form

$$
\mathrm{e}^{\int m^{\text {bos }}(z)-\operatorname{deg}^{\text {bos }}(z)+n^{\text {bos }}(z)} P\left(\tilde{n}^{\text {ferm }}\right), m \in K_{M}, n \in K_{M}^{\vee}
$$

to the fields of the form

$$
\mathrm{e}^{\int m^{\text {bos }}(z)-\operatorname{deg}^{\text {bos }}(z)+n^{\text {bos }}(z)} P\left(\tilde{n}^{\text {ferm }}\right), m \in K_{N}^{\vee}, n \in K_{N}
$$

modulo the image of $D_{f, g}$. Presumably, this is what was accomplished ad hoc in $\mathrm{Kra}$ by using the classification of invertible potentials.

\section{Unification of Berglund-HÜBSCH AND Batyrev-Borisov CONSTRUCTIONS}

In this section we describe the unified setting of duality that includes both Batyrev-Borisov and Berglund-Hübsch as special cases. We comment on necessary combinatorial conditions.

7.1. Combinatorial conditions. We start with dual lattices $M$ and $N$ with chosen elements deg and $\mathrm{deg}^{\vee}$ in them and collections of elements $\Delta \subset M$ and $\Delta^{\vee} \subset N$. We first require that for all $m \in \Delta$, $n \in \Delta^{\vee}$ there holds $\operatorname{deg} \cdot n=m \cdot \operatorname{deg}^{\vee}=1$ and $m \cdot n \geq 0$. We define the cones $K_{M}$ and $K_{N}$ as being spanned by $\Delta$ and $\Delta^{\vee}$ respectively. We abuse the notation to use the same symbols for the sets of the lattice points in these cones. We assume that $K_{M}$ and $K_{N}$ are of full dimension. Their duals are denoted by $K_{N}^{\vee}$ and $K_{M}^{\vee}$ and we have

$$
K_{N}^{\vee} \supseteq K_{M}, K_{M}^{\vee} \supseteq K_{N}
$$

We also consider the coefficient functions $f: \Delta \rightarrow \mathbb{C}$ and $g: \Delta^{\vee} \rightarrow \mathbb{C}$. 
Definition 7.1.1. The data $\left(M, N, \Delta, \Delta^{\vee}\right.$, deg, deg $\left.{ }^{\vee}\right)$ above are said to give the discrete data of toric mirror symmetry if the following two (dual) conditions are satisfied for generic $(f, g)$. First, for every onedimensional face of $K_{N}^{\vee}$ there exists a nonzero lattice point $v$ on it and elements $P_{n} \in \mathbb{C}[M]$ such that

$$
[v]=\sum_{n \in \Delta^{\vee}}\left(P_{n} \sum_{m \in \Delta} f(m)(m \cdot n)[m]\right)
$$

where $P_{n}$ have the additional property that all of their monomials $[w]$ satisfy $w \cdot n \geq-1$ and $w \cdot \hat{n} \geq 0$ for all $\hat{n} \in \Delta$ for $\hat{n} \neq n$. Second, we assume that the dual statement for $K_{M}^{\vee}$ and $g$ holds.

Remark 7.1.2. We observe that the above conditions are precisely the ones we have proved in Proposition 5.2.1 for the Berglund-Hübsch case, together with the dual statement. These conditions also hold for the Batyrev-Borisov case where they amount to Batyrev's nondegeneracy condition from [Ba1.

We will give a simple restatement of the condition of Definition 7.1.1 in terms of the homogeneous coordinate rings of [Co]. For the cone $K_{N}^{\vee}$ and the set $\Delta^{\vee}$ consider the polynomial ring $\mathbb{C}\left[x_{i}\right]$ with the number of variables equal to the number of elements in $\Delta^{\vee}$. One can encode an element of $K_{N}^{\vee}$ by its pairings with elements of $\Delta^{\vee}$. Conversely, a collection of pairings with elements of $\Delta^{\vee}$ gives an element of $K_{N}^{\vee}$ provided all the pairings are nonnegative and satisfy some linear relations and/or congruences. This easy observation identifies $\mathbb{C}\left[K_{N}^{\vee}\right]$ as the invariant subring of $\mathbb{C}\left[x_{1}, \ldots, x_{\sharp\left(\Delta^{\vee}\right)}\right]$ under the diagonal action of some abelian algebraic group $G$ of dimension $\sharp\left(\Delta^{\vee}\right)-\operatorname{rk} M$.

Proposition 7.1.3. Define the potential $W=\sum_{m \in \Delta} f(m) \prod_{n \in \Delta} x_{n}^{m \cdot n}$. Consider the Jacobian ideal Jac $(W)$ generated by the partial derivatives of $W$. Then the first condition of Definition 7.1.1 is equivalent to the condition that

$$
\mathbb{C}\left[K_{N}^{\vee}\right] /\left(\mathbb{C}\left[K_{N}^{\vee}\right] \cap \operatorname{Jac}(W)\right)
$$

is finite-dimensional.

Proof. Suppose that the above quotient is finite-dimensional. It is clearly $M$-graded, which means that only a finite number of gradings occur. Thus for a ray of $K_{N}^{\vee}$ there is an element $v$ on it such that $\prod_{n \in \Delta^{\vee}} x_{n}^{v \cdot n} \in \operatorname{Jac}(w)$. The rest of the argument follows the proof of Proposition 5.2.1. In the other direction, the condition of Definition 7.1 .1 is equivalent to the property that $\mathbb{C}\left[K_{N}^{\vee}\right] \cap \operatorname{Jac}(W)$ contains elements $[v]$, at least one for each ray. Since it is clearly an ideal of $\mathbb{C}\left[K_{N}^{\vee}\right]$, this implies that the quotient is finite-dimensional. 
Remark 7.1.4. The following combinatorial condition is necessary, but is perhaps not sufficient. For every facet $\theta$ of $K_{N}$ there exists an element $m \in \Delta$ and an element $n \in \theta$ such that $m \cdot n \leq 1$ and $m \cdot \hat{n}=0$ for all other $\hat{n} \in \theta$. Indeed, one picks $m$ and $n$ in Definition 7.1.1 that contribute nontrivially to the graded piece $v$ and use $\theta$ which is dual to the ray through $v$. Of course, one also needs to impose the dual condition, which does not appear to follow directly from the original condition.

7.2. Vertex algebras of toric mirror symmetry. In the setting of the previous subsection, we can define vertex algebras $V_{f, g}$ and their chiral rings, for the generic choices of $f$ and $g$.

Definition 7.2.1. Let $\left(M, N, \Delta, \Delta^{\vee}, \operatorname{deg}, \operatorname{deg}^{\vee}\right)$ be the discrete data of toric mirror symmetry. Let $f$ and $g$ be coefficient functions. We define the vertex algebra $V_{f, g}$ as the cohomology of the lattice vertex algebra Fock $_{M \oplus N}$ by the differential

$D_{f, g}:=\operatorname{Res}_{z=0}\left(\sum_{m \in \Delta} f(m) m^{f e r m}(z) \mathrm{e}^{\int m^{b o s}(z)}+\sum_{n \in \Delta^{\vee}} g(n) n^{f e r m}(z) \mathrm{e}^{\int n^{b o s}(z)}\right)$.

Recall the definition of four subcomplexes of Fock $_{M \oplus N}$ given in Proposition 6.1.1.

Theorem 7.2.2. If $f$ and $g$ are generic, then the vertex algebra $V_{f, g}$ is of $\sigma$-model type. Its $A$ ring may be calculated either as the cohomology of the subcomplex $\mathcal{C}_{K_{N}^{\vee}, K_{N}-\operatorname{deg}^{\vee}, M}$ or the cohomology of $\mathcal{C}_{K_{M}, K_{M}^{\vee}-\operatorname{deg}^{\vee}, M}$. Its $B$ ring may be calculated either as cohomology of $\mathcal{C}_{K_{N}^{\vee}-\operatorname{deg}, K_{N}, N}$ or the cohomology of $\mathcal{C}_{K_{M}-\mathrm{deg}, K_{M}^{\vee}, N}$. There is a spectral flow isomorphism (as vector spaces) between the $A$ ring and the $B$ ring, given by considering the appropriate terms of the OPEs with the fields $\mathrm{SF}_{ \pm}(z)$ considered in Proposition 5.3.1.

Proof. We observe that the proof of Theorem 6.2.1 goes through in this more general situation. Specifically, the condition of Definition 5.2.1 assures that the proof of the Key Lemma still works. Then one needs to further assume that $f$ and $g$ are strongly nondegenerate in the sense of [Bo3], to show that $V_{f, g}$ is of $\sigma$-model type and to give one calculation of each of the chiral rings. Another calculation is obtained via the spectral flow isomorphism, as in the proof of Theorem 6.2.1.

\section{Open PROBLEMS}

In this section we comment on the open questions related to our construction. 
Classification. Similar to the Batyrev-Borisov situation, one may hope to classify all $\Delta$ and $\Delta^{v}$ which satisfy the conditions of Definition 7.1.1, in the case of $\operatorname{deg} \cdot \operatorname{deg}^{\vee}=1$ and $\hat{c}=3$. As in KreS2, this amounts to a classification of certain four-dimensional polytopes, although reflexivity condition is now somewhat relaxed. Given that the number of nonequivalent dimension four reflexive polytopes is around $5 \cdot 10^{8}$ this can be potentially an arduous task. The first step in this direction would be a reformulation of Definition 7.1.1 in purely combinatorial terms which do not involve the coefficient functions.

Frobenius algebra structure. Our construction endows the $A$ and $B$ rings of Berglund-Hübsch mirror symmetry with the structure of Frobenius algebra, by evaluating against the top class (a generator of bidegree $(\hat{c}, \hat{c}))$.

It is important to check that the product in our rings coincides with the known constructions whenever applicable. While this should be reasonably straightforward for Berglund-Hübsch models, there appear to be serious difficulties in the general setting of Definition 7.1.1. At the moment, we can not even prove that the corresponding Hodge diamond is symmetric. There are also some technical difficulties in comparing the usual Hessian (used in Berglund-Hübsch construction) with the logarithmic Hessian (for Batyrev-Borisov construction). It is conceivable that Definition 7.1.1 may need to be somehow augmented.

It is an open problem in Batyrev-Borisov setting to define the product on the chiral rings without the use of vertex algebras. While the Berglund-Hübsch case might be a bit easier, it is far from obvious. In particular there is a general procedure of orbifoldizing Frobenius algebras due to Kaufmann, which often significantly narrows down the space of possible products, see Kau3. The first step in this direction would be an understanding of the analogs of the subrings of Remark 3.1 .6 .

Elliptic genera of Berglund-Hübsch models. The elliptic genera of Berglund-Hübsch models have been calculated in [BeHe] where it was observed that they satisfy the expected duality. This invariant should encode the double-graded superdimension of $V_{f, g}$, which will naturally behave well under passing to the mirror. To prove the comparison, one might be able to use the method of [BoL], which appears to be applicable to the Berglund-Hübsch setting.

Comments on geometry. The results of Section 3 may be useful in the setting of [ChR], where the Landau-Ginzburg model is compared to the orbifold theory. It is plausible that the spaces of the orbifold 
theory are formally obtained from those of LG theory by replacing one of the semigroup rings by a partial semigroup ring, which corresponds to the fan of the ambient toric variety.

\section{REFERENCES}

[Ba1] V. Batyrev, Variations of the mixed Hodge structure of affine hypersurfaces in algebraic tori, Duke Math. J. 69 (1993), no. 2, 349-409.

[Ba2] V. Batyrev, Dual polyhedra and mirror symmetry for Calabi-Yau hypersurfaces in toric varieties, J. Algebraic Geom. 3 (1994), no. 3, 493-535.

[BaN] V. Batyrev, B. Nill, Combinatorial aspects of mirror symmetry. Integer points in polyhedra. Geometry, number theory, representation theory, algebra, optimization, statistics. 35-66, Contemp. Math., 452, Amer. Math. Soc., Providence, RI, 2008.

[BeHe] P. Berglund, M. Henningson, Landau-Ginzburg orbifolds, mirror symmetry and the elliptic genus. Nuclear Phys. B 433 (1995), no. 2, 311-332.

[BeH] P. Berglund, T. Hübsch, A Generalized Construction of Mirror Manifolds. Nuclear Physics B, 393 (1993), no. 1-2, 377-391.

[Bo1] L. Borisov, Towards the Mirror Symmetry for Calabi-Yau Complete intersections in Gorenstein Toric Fano Varieties. Preprint arXiv:alg-geom/9310001.

[Bo2] L. Borisov, Vertex algebras and mirror symmetry. Comm. Math. Phys. 215 (2001), no. 3, 517-557.

[Bo3] L. Borisov, Chiral rings of vertex algebras of mirror symmetry. Math. Z. 248 (2004), no. 3, 567-591.

[BoM] L. Borisov, A. Mavlyutov, String cohomology of Calabi-Yau hypersurfaces via mirror symmetry. Adv. Math. 180 (2003), no. 1, 355-390.

[BoL] L. Borisov, A. Libgober, Elliptic genera of toric varieties and applications to mirror symmetry. Invent. Math. 140 (2000), no. 2, 453-485.

[CaOGP] P. Candelas, X. de la Ossa, P. Green, L. Parkes, An exactly soluble superconformal theory from a mirror pair of Calabi-Yau manifolds. Phys. Lett. B 258 (1991), no. 1-2, 118-126.

[ChR] A. Chiodo, Y. Ruan, $L G / C Y$ correspondence: the state space isomorphism. Preprint arXiv:0908.0908.

[Co] D. Cox, The homogeneous coordinate ring of a toric variety. J. Algebraic Geom. 4 (1995), no. 1, 17-50.

[Kac] V. Kac, Vertex algebras for beginners. Second edition. University Lecture Series, 10. American Mathematical Society, Providence, RI, 1998.

[Kau1] R. Kaufmann, Orbifold Frobenius algebras, cobordisms and monodromies. Orbifolds in mathematics and physics (Madison, WI, 2001), 135-161, Contemp. Math., 310, Amer. Math. Soc., Providence, RI, 2002.

[Kau2] R. Kaufmann, Orbifolding Frobenius algebras. Internat. J. Math. 14 (2003), no. 6, 573-617.

[Kau3] R. Kaufmann, Singularities with symmetries, orbifold Frobenius algebras and mirror symmetry. Gromov-Witten theory of spin curves and orbifolds, 67-116, Contemp. Math., 403, Amer. Math. Soc., Providence, RI, 2006 . 
[Kra] M. Krawitz, FJRW rings and Landau-Ginzburg Mirror Symmetry. Preprint arXiv:0906.0796.

[KreS1] M. Kreuzer, H. Skarke, On the classification of quasihomogeneous functions. Comm. Math. Phys. 150 (1992), no. 1, 137-147.

[KreS2] M. Kreuzer, H. Skarke, Complete classification of reflexive polyhedra in four dimensions. Adv. Theor. Math. Phys. 4 (2000), no. 6, 1209-1230.

[LeVW] W. Lerche, C. Vafa, N. P. Warner, Chiral rings in $N=2$ superconformal theories. Nuclear Phys. B 324 (1989), no. 2, 427-474.

[MSV] F. Malikov, V. Schechtman, A. Vaintrob, Chiral de Rham complex, Comm. Math. Phys. 204 (1999), no. 2, 439-473.

Mathematics Department, Rutgers University, 110 Frelinghuysen RD, PiscataWAy, NJ 08854, EMAIL:Borisov@Math.RUTGERS.EDU 